Hydroécol. Appl. (1990) 1/2, pp. 55-89

\title{
Evaluation quantitative de la biomasse végétale en Durance à l'aval du barrage de Serre-Ponçon.
}

\author{
M. Khalanski ${ }^{(1)}$, M. Bonnet et A. Grégoire ${ }^{(2)}$. \\ (1) Electricité de France - Direction des Etudes ot Recherches, 6, Quai Watior 78401 Chatou \\ Cedex. France. \\ (2) Electricité de France - Direction de l'Equipement, Service IDE. 22-30 avenue de Wagram. \\ 75382 Paris cedex 08. France.
}

Résumé. - La quantité de végétaux macrophytes a été évaluée en poids frais et en poids sec dans un tronçon de la Durance, une rivière du sud de la France. Le tronçon étudié se situe à l'aval de Serre-Ponçon, la plus grande retenue hydroélectrique française; il comporte le bassin de compensation d'Espinasses et la Durance sur environ $3 \mathrm{~km}$ jusqu'au pont de Rochebrune.

Dans les rivières à débit régulé où les fonds sont en partie envasés, se développe généralement une abondante végétation composée de plantes supérieures et d'algues, c'est le cas de la Durance en aval de Serre-Ponçon. Le débit minimum maintenu en permanence dans la rivière est de $1,5 \mathrm{~m}^{3} / \mathrm{s}$.

Des mesures ont été réalisées en 1985, au cours de l'été et de l'automne, en vue de déterminer quantitativement la biomasse des macrophytes, d'évaluer leur charge en phosphore et de recueillir des données sur leur répartition en fonction des facteurs d'environnement. Des données de qualité d'eau ont d'autre part été collectées pendant la période de mesures afin d'apprécier l'incidence de la végétation macrophyte sur le bilan d'oxygène dissous de la rivière.

Dans le bassin de compensation d'Espinasses d'une superficie de 112,5 ha, avec une profondeur moyenne de 5,4 $\mathrm{m}$, la biomasse végétale maximum atteint en fin septembre 95 tonnes en poids sec soit $844 \mathrm{~kg} / \mathrm{ha}$; elle contient $86 \mathrm{~kg}$ de phosphore.

Le tronçon de Durance étudié, long de $2,8 \mathrm{~km}$, d'une superficie de $12,3 \mathrm{ha}$, a une biomasse maximum de 29 tonnes en poids sec soit $2300 \mathrm{~kg} / \mathrm{ha}$; elle contient $45 \mathrm{~kg}$ de phosphore.

Les Characées représentent à la fin du mois d'août environ $90 \%$ de la biomasse dans le bassin d'Espinasses et $42 \%$ dans la rivière. A la fin du mois de septembre les Characées représentent $98 \%$ de la biomasse dans le bassin et $83 \%$ dans la Durance. 
Le reste de la végétation est constitué presque exclusivement par des Potamogétonacées: Groenlandia densa (Potamogeton densus) et Potamogeton pectinatus.

Les algues vertes des genres Cladophora et Spirogyra constituent une fraction mineure de la biomasse totale, avec un maximum de $5 \%$ à la fin novembre.

Le phosphore apporté par l'eau de Serre-Ponçon, bien qu'en faible concentration, n'est pas limitant pour la croissance végétale.

Les mesures en continu de $\mathrm{pH}$ et d'oxygène dissous effectués sur ce tronçon mettent en évidence l'influence de la photosynthèse et de la respiration des végétaux. En septembre, les fluctuations journalières atteignent 0,6 unité $\mathrm{pH}$ et $3,0 \mathrm{mg} / \mathrm{l}$ pour l'oxygène dissous. La teneur en oxygène dissous est toutefois restée supérieure ou égale à $7,8 \mathrm{mg} / \mathrm{l}$ en moyenne mensuelle de juin à novembre, le minimum n'étant pas inférieur à $6,0 \mathrm{mg} / \mathrm{l}$.

Summary. - The quantity of macrophyte plants has been evaluated in terms of fresh and dry weight in a section of the Durance river in the South of France. The section under study is downstream of Serre-Ponçon, the largest hydro-electric reservoir in France : it comprises the Espinasses compensation basin and the Durance along approximately $3 \mathrm{~km}$ to the Rochebrune bridge.

In regulated flow rivers with partly muddy beds, abundant plant life generally develops. This is true of the Durance downstream of Serre-Ponçon. The minimum rate of flow which is continuously maintained in the river is $1.5 \mathrm{~m}^{3}$ per sec.

Readings were made in 1985 in the summer and autumn to determine the quantity of macrophyte biomass, evaluate its phosphorus content and collect data on its distribution with respect to environmental factors. Data relating to water quality were also collected during the measurement period to asses the effect of macrophyte plants on the dissolved oxygen balance of the river.

In the Espinasses compensation basin, area 112.5 hectares and average depth $5.4 \mathrm{~m}$, the maximum plant biomass reached end-september was 95 tonnes dry weight, ie, $844 \mathrm{~kg}$ per hectare. It contained $86 \mathrm{~kg}$ of phosphorus.

The Durance section under study, length $2.8 \mathrm{~km}$ and area 12.3 hectares, has a maximum biomass of 29 tonnes dry weight, ie, $2300 \mathrm{~kg}$ per hectare and contains $45 \mathrm{~kg}$ of phosphorus. The Characeae represent approximately $90 \%$ of biomass at the end of August in the Espinasses basin and $42 \%$ in the river. At the end of septembre, the Characeae represent $98 \%$ of biomass in the basin and $83 \%$ in the Durance. Other plants are basically Potamogetonaceae : Groenlandia densa (Potamogeton densus) and Potamogeton pectinatus.

Cladophora and Spirogyra green algae are minor fraction of total biomass with a maximum of $5 \%$ end-November.

Although of low content, the phosphorus in the water of Serre-Ponçon is not a limiting factor for plant growth.

On-going $\mathrm{pH}$ and dissolved oxygen measurements conducted in this section reveal the influence of photosynthesis and plant respiration. In september, fluctuations in $\mathrm{pH}$ reach 0,6 and $3.0 \mathrm{ppm}$ for dissolved oxygen. The dissolved oxygen balance nevertheless remains higher than or equal to $7.9 \mathrm{ppm}$ on average from June to November; the minimum is not lower than $6.0 \mathrm{ppm}$. 


\section{1 - INTRODUCTION.}

Dans les rivières à débit réservé se développe fréquemment une abondante végétation composée de plantes supérieures et d'algues; c'est le cas de la Durance à l'aval de Serre-Ponçon.

Ce phénomène a été notamment observé à l'aval de barrages sur le Lot et la Truyère par Décamps et al (1975), sur la Dordogne à l'aval d'Argentat (Rapports Agence de Bassin 1974 - et Cemagref - 1982), sur le Verdon à l'aval de Gréoux par Grégoire et al. (1975).

L'origine de la végétation réside dans trois facteurs principaux : sédimentologique, bathymétrique et trophique.

La présence permanente d'une couche de sédiments fins, est indispensable au développement des végétaux à racines.

La faible profondeur favorise le transfert du rayonnement solaire dans la masse d'eau; il faut noter que la haute Durance présente un niveau élevé d'ensoleillement.

Les apports de nutriments, principalement de phosphore, conditionnent la croissance végétale.

Les conséquences de l'implantation des macrophytes pour l'écosystème durancien sont diverses:

- la végétation fixe les sédiments par son système racinaire et contribue à maintenir et à accentuer l'envasement des fonds;
- elle influe sur la qualité de l'eau, notamment le bilan d'oxygène dissous et de nutriments;

- elle apporte une ressource trophique importante pour certaines espèces de poissons par la production d'invertébrés qui lui sont associés (insectes, crustacés, mollusques, Hirudinés...);

- elle sert d'abri aux juvéniles de certaines espèces de poissons et à nombre d'invertébrés benthiques.

Les campagnes de mesures qui font l'objet de ce rapport s'inscrivent dans une étude pluridisciplinaire visant à évaluer l'impact du débit réservé sur l'écosystème durancien entre Espinasses et la Saulce.

Quatre campagnes de mesures ont été réalisées de août 1985 à novembre 1985 avec la collaboration de I'Université de Provence et le concours du Bureau d'Etudes Aquascop.

Elles avaient un triple objectif :

- déterminer quantitativement la biomasse maximale des macrophytes dans le bassin de compensation d'Espinasses et dans la Durance jusqu'au pont de Rochebrune,

- recueillir des données sur l'écologie des macrophytes en terme de répartition en fonction des facteurs d'environnement,

- préciser les relations entre la végétation et la qualité de l'eau, particulièrement les apports en phosphore et le bilan d'oxygène dissous.

L'ensemble des données collectées figure dans les annexes du rapport établi par M. Khalanski et al. (1987). 


\section{2 - LIEU D'ETUDE.}

Rivière des Alpes du Sud, la Durance, affluent en rive gauche du Rhône, prend sa source au Mont Genèvre (2 $300 \mathrm{~m})$. Dans le tronçon étudié son module est de $80 \mathrm{~m}^{3} / \mathrm{s}$.

A l'amont de Serre Ponçon, la Durance longue de $75 \mathrm{~km}$ a gardé toutes ses caractéristiques de cours d'eau de montagne à régime nival: vitesse de courant rapide, substrat rocheux et crue de printemps. Le lit est libre de toute végétation macrophytique.

La retenue de Serre Ponçon, située en aval d'Embrun, avec une capacité de 1,3 milliards de $\mathrm{m}^{3}$ et une profondeur de $120 \mathrm{~m}$ est la plus grande retenue de France.

A l'aval du barrage, l'eau turbinée transite par le bassin de compensation d'Espinasses puis est dérivée en plus grande partie par le canal de Curbans jusqu'à la retenue de la Saulce, située $11 \mathrm{~km}$ à l'aval d'Espinasses. Le débit réservé dans le lit naturel de la Durance au barrage d'Espinasses est en permanence de $1,5 \mathrm{~m}^{3} / \mathrm{s}$. Le débit varie sur le tronçon du fait d'apports ponctuels par des affluents ou d'apports diffus.

Les campagnes sur le terrain ont été menées à l'aval immédiat de la retenue, dans le bassin de compensation d'Espinasses et dans la partie de Durance court-circuitée comprise entre le barrage mobile d'Espinasses et Tallard (figures 1 et 2).

\section{- Le bassin de compensation d'Espinasses.}

Ce petit bassin d'une capacité de 6 à 11 millions de $\mathrm{m}^{3}$ et d'une superficie d'environ 120 ha régularise le débit d'écoulement de l'eau à l'aval de l'usine hydroélectrique de Serre-Ponçon. Ses dimensions sont environ de $2900 \mathrm{~m}$ en longueur et $400 \mathrm{~m}$ en largeur. Du fait de son rôle régulateur il subit un marnage journalier dont l'amplitude maximum atteint $4 \mathrm{~m}$ entre les côtes 653,50 et 657,50 .

Volume et superficie du plan d'eau en fonction de la côte.

\begin{tabular}{|l|c|c|}
\hline \multicolumn{1}{|c|}{ cote $(\mathrm{m})$} & $\begin{array}{c}\text { superficie } \\
\text { (ha) }\end{array}$ & $\begin{array}{c}\text { volume } \\
\text { (millions de } \mathrm{m}^{3} \text { ) }\end{array}$ \\
\hline 653,5 (minj- & 118,5 & 6,25 \\
mum) & - & 6,75 \\
654 & 119,0 & 8,00 \\
655 & 120,4 & - \\
656 & 121,7 & - \\
657 & 122,3 & 11,00 \\
657,5 & & \\
\hline
\end{tabular}

Les relevés bathymétriques réalisés au cours de nos campagnes de mesures indiquent une profondeur maximum de $7 \mathrm{~m}$.

\section{- Le tronçon de rivière court-circuité.}

La morphologie de la rivière dans le secteur étudié est caractérisé par une succession de radiers où l'eau est courante et peu profonde (moins de $50 \mathrm{~cm}$ ) et de "pools", zones plus profondes, dans lesquelles la vitesse est réduite. Dans ces secteurs le lit de la rivière s'élargit et atteint par exemple 
Biomasse végétale en Durance à l'aval de Serre-Ponçon
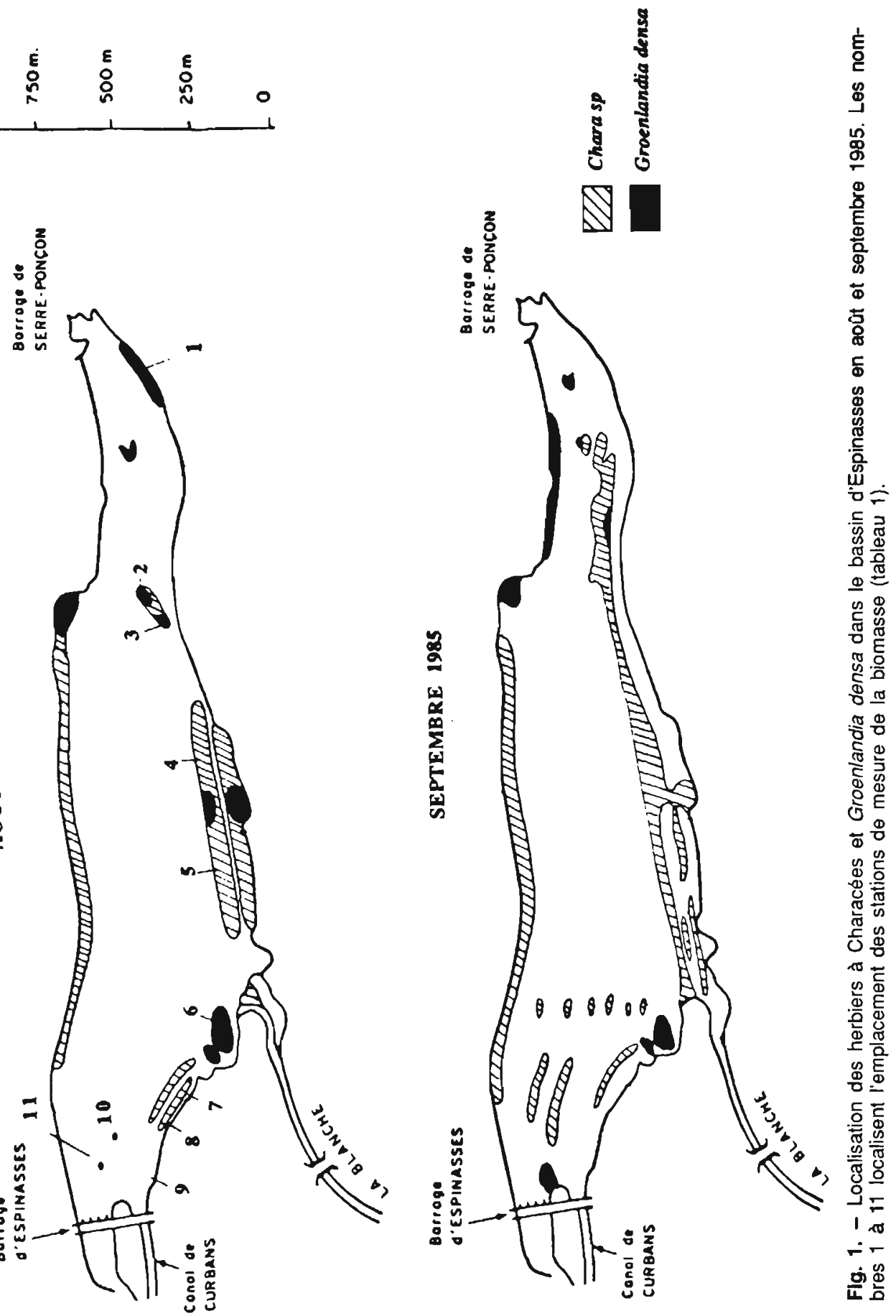
100 mètres dans le grand pool à l'amont du pont de Rochebrune.

Une partie du lit de la rivière est recouverte d'une couche de vase dont l'épaisseur varie de quelques $\mathrm{cm}$ dans les zones courantes à plus de $2 \mathrm{~m}$ dans le pool à l'amont du pont de Rochebrune.

Le fond originel constitué de galets et gros blocs n'apparaît plus qu'au centre du lit dans les zones de "rapides".

La végétation aquatique, présente presque toute l'année, accentue le processus de sédimentation par réduction de la vitesse du courant et surtout par piégeage mécanique des particules en suspension particulièrement abondantes en période d'orages.

\section{3 - METHODOLOGIE.}

\subsection{Cartographie et évaluation de la biomasse végétale.}

L'étude de la végétation a été réalisée à l'aide de relevés sur le terrain et de photographies aériennes.

\section{Relevés sur le terrain.}

\section{Calendrier des campagnes.}

Les prospections in situ ont été effectuées aux périodes généralement les plus propices à la prolifération des végétaux :

du $26 / 08$ au $30 / 08 / 85$

du 23/09 au 27/09/85

du 25/11 au 28/11/85.
Choix des stations.

- Dans le bassin de compensation, 6 transects espacés de $500 \mathrm{~m}$ environ ont été prospectés.

- Dans le tronçon court-circuité, l'effort a été particulièrement accentué entre le pont d'Espinasses et le pont de Rochebrune, soit sur $2800 \mathrm{~m}$ de rivière, où 30 transects et stations y ont été prospectés (fig. 2).

Les transects sont rapprochés de quelques mètres seulement dans les zones où interviennent des changements morphologiques du cours d'eau, et au contraire plus espacés $(200 \mathrm{~m})$ lorsque les conditions du milieu apparaissent homogènes.

Enfin, quatre autres transects, répartis plus en aval entre le pont de Rochebrune et le bassin de la Saulce, ont été prospectés (fig. 3). Sur ce tronçon se trouvent une gravière en amont du pont de l'Archidiacre et divers rejets d'eaux usées; notamment par la Luye qui évacue les eaux usées de la ville de Gap après épuration.

\section{Mesures en chaque transect.}

a) Identification des espèces végétales présentes et évaluation de l'importance relative des différentes espèces le long du transect.

b) Prélèvements d'échantillons de végétation (cadrat de $0,25 \mathrm{~m}^{2}$ ) répartis le long du transect en vue de la détermination du poids frais, du poids sec et de la teneur en phosphore des tissus après minéralisation selon la méthode décrite en annexe 2 du rapport Khalanski et al., 1987. 

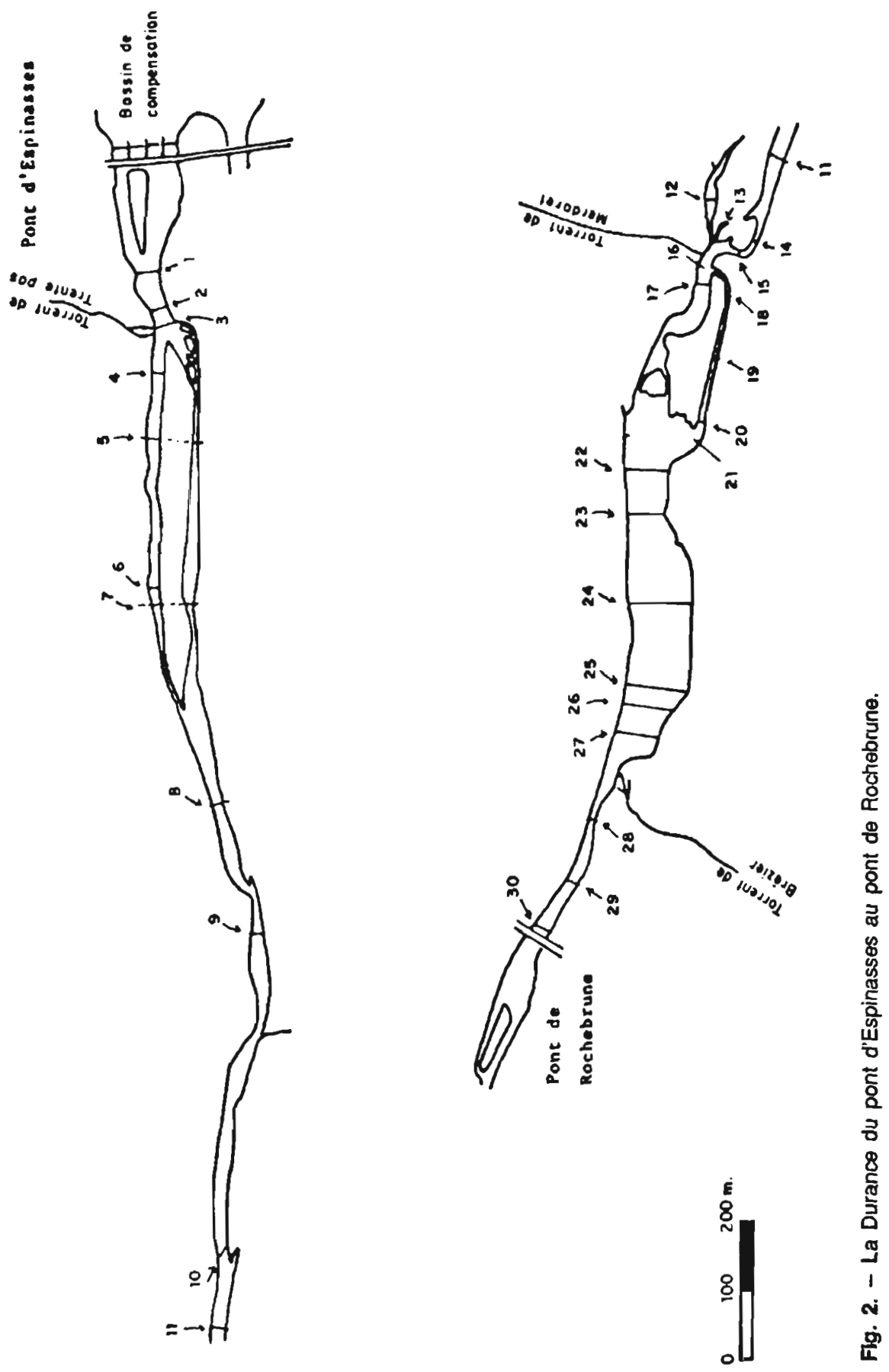


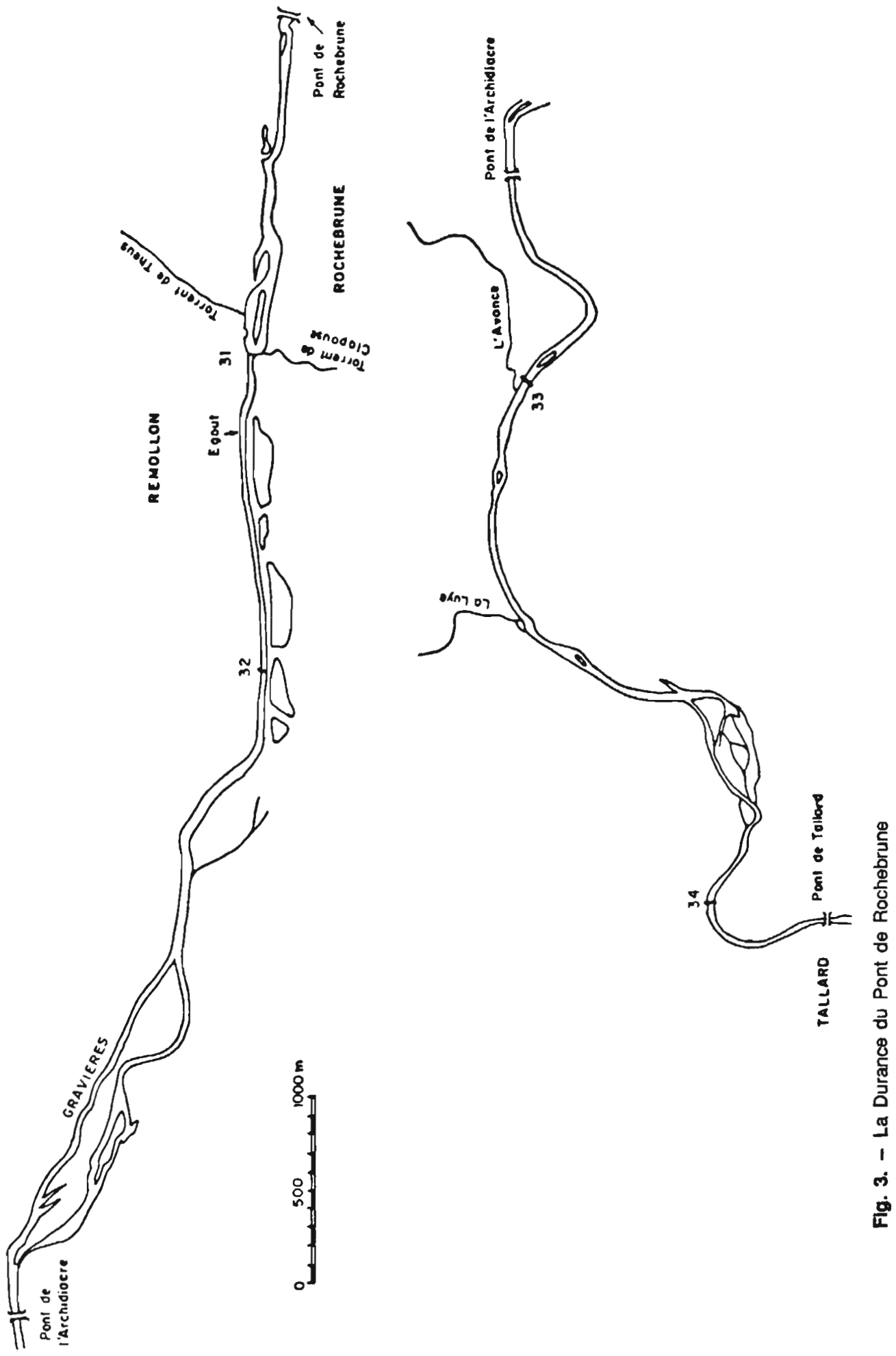


c) Calcul de la biomasse moyenne sur chaque transect à partir du poids moyen (en $\mathrm{g} / \mathrm{m}^{2}$ ) dans les cadrats et du pourcentage d'occupation par chaque espèce.

\section{Observations sub-aquatiques.}

L'observation de la répartition de la végétation immergée dans les plans d'eau profonds (bassin de compensation d'Espinasses et pool de Rochebrune) a été effectuée par AQUASCOP au cours de plongées en scaphandre autonome en septembre et novembre 1985.

\section{Photographies aériennes.}

Cette étude in situ a été complétée par la prise de photographies aériennes par hélicoptère, en octobre 1985, permettant de réaliser une cartographie de la végétation sur l'ensemble du secteur d'étude.

\subsection{Recueil de données complé- mentaires.}

- Parallèlement à l'évaluation de biomasse, des dosages d'azote et phosphore ont été effectués sur des échantillons d'eau prélevés lors des trois campagnes en 1985 entre Espinasses et Tallard. Quelques analyses avaient été réalisées en juillet 1985 .

- Des dosages de matières en suspension totales ont été effectués en août et septembre 1985.

- Deux stations automatiques mesurant séquentiellement en continu la température, le $\mathrm{pH}$, la conductivité et l'oxygène dissous ont été installées au pont de Rochebrune et au pont de l'Archidiacre à partir de juin 1985. Les données obtenues permettent de suivre l'évolution de ces paramètres au cours de la journée depuis cette date.

\section{4 - LOCALISATION DES HERBIERS, SURFACE DE LA COUVERTURE VÉGÉTALE, BIOMASSE ET CHARGE EN PHOSPHORE}

\subsection{Le bassin de compensation d'Espinasses.}

\section{Répartition verticale.}

La végétation dans le bassin est constituée essentiellement de Characées et Groenlandia densa sous forme de vastes tapis pouvant atteindre une hauteur de $2 \mathrm{~m}$. On observe également quelques spirogyres à l'entrée de la Blanche. Les spirogyres se trouvent à la fois en épiphytes sur les Chara et les Groenlandia et sur le fond nu.

La répartition des herbiers en fonction de la profondeur montre qu'audelà de $2,50 \mathrm{~m}$ de profondeur les G. densa disparaissent. Par contre les Characées couvrent le fond jusqu'à une profondeur de $4,5 \mathrm{~m}$ et on trouve des spirogyres jusqu'à $7 \mathrm{~m}$.

Evolution de la couverture végétale d'août à novembre 1985.

- En août, les $G$. densa occupent une surface de $15000 \mathrm{~m}^{2}$ environ, soit un peu plus de $1 \%$ de la super- 
Tableau 1. - Mesures de biomasses effectuées en août 1985 en 10 stations dans le bassin d'Espinasses. Les stations sont localisées sur la figure 1.

\begin{tabular}{|c|c|c|c|c|}
\hline Stations & Espèce & Profondeur & $\begin{array}{c}\text { Biomasse } \\
\text { en g de poids sec } \\
\text { par } \mathrm{m}^{2}\end{array}$ & $\begin{array}{c}\text { Surface } \\
\text { de la tache en m2 }\end{array}$ \\
\hline 1 & $\begin{array}{c}\text { G. densa } \\
\text { Chara } s p\end{array}$ & $\begin{array}{c}\text { de } 2 \mathrm{~m} \text { à } 4 \mathrm{~m} \\
\text { de } 2 \mathrm{~m} \text { à } 4 \mathrm{~m}\end{array}$ & 663,8 & 3000 \\
\hline 2 & G. densa & de $1 \mathrm{~m}$ à $2,30 \mathrm{~m}$ & 362,8 & 3000 \\
\hline $3-4 \cdot 5$ & Chara sp & de $0,6 \mathrm{~m}$ à $1,0 \mathrm{~m}$ & 2101,4 & 21000 \\
\hline 6 & G. densa & $1,0 \mathrm{~m}$ & 429,3 & 9100 \\
\hline 7 & Chara $s p$ & au bord & 1745,6 & 9900 \\
\hline 8 & Chara $s p$ & au bord & 1040,1 & 9900 \\
\hline 9 & Chara $s p$ & au bord & 911,5 & $<10$ \\
\hline 10 & G. densa & $1,5 \mathrm{~m}$ à $2,0 \mathrm{~m}$ & 271,8 & 10 \\
\hline 11 & G. densa & $1,5 \mathrm{~m}$ à $2,0 \mathrm{~m}$ & 359,0 & 24 \\
\hline
\end{tabular}

ficie du bassin. Les Characées, capables de coloniser de plus grandes profondeurs, recouvrent $3 \%$ de la surface du bassin (fig. 1).

- En septembre, la végétation occupe $10 \%$ de la superficie du bassin, soit plus du double de la surface observée en août. Cette augmentation est due au développement des Characées (fig. 1).

- En novembre, compte tenu des très mauvaises conditions climatiques, l'analyse de la végétation du bassin n'a pu être effectuée que par des sondages ponctuels. II semble que $G$. densa ait totalement disparu; on en retrouve près des berges dans un état de décomposition avancée. Les Characées par contre occupent toujours le fond du bassin et paraissent en bon état.

\section{Estimation de la biomasse.}

On constate, à partir des nombreux prélèvements effectués en août, que les densités (exprimées en $g$ de poids sec par $\mathrm{m}^{2}$ ) sont élevées, surtout près des berges, c'est-à-dire dans les prélèvements effectués à faible profondeur (ct. tableau 1).

On ne possède, pour la campagne de septembre, que deux valeurs de biomasse obtenues sur des cadrats :

G. densa: $60,4 \mathrm{~g} \mathrm{PS} / \mathrm{m}^{2}$ - Characées: $1164 \mathrm{~g} \mathrm{PS} / \mathrm{m}^{2}$.

La biomasse par $\mathrm{m}^{2}$ des $G$. densa est faible si on la compare à celle du mois d'août (cette diminution est également observée dans la rivière).

Le tableau 2 présente les valeurs de biomasse totale et relative en $\mathrm{kg}$ 
Tableau 2 - Evaluation de la biomasse végétale dans le bassin d'Espinasses.

\begin{tabular}{|c|c|c|c|c|c|c|}
\cline { 2 - 7 } \multicolumn{1}{c|}{} & \multicolumn{3}{c|}{$\begin{array}{c}\text { Biomasse totale en } \mathrm{kg} \text { de } \\
\text { matières sèches }\end{array}$} & \multicolumn{3}{c|}{$\begin{array}{c}\text { Biomasse en kg de matières } \\
\text { sèches par ha }\end{array}$} \\
\hline Période & G. densa & Chara. & Total & G. densa & Chara. & Total \\
\hline $\begin{array}{c}26-30 \text { août } \\
1985\end{array}$ & 7000 & 62923 & $\# 70000$ & 62 & 559 & 622 \\
\hline $\begin{array}{c}23-27 \\
\text { sept. } 1985\end{array}$ & 1812 & 93152 & $\# 95000$ & 16 & 828 & 844 \\
\hline
\end{tabular}

par ha pour l'ensemble du bassin d'Espinasses.

\subsection{La Durance à l'aval du bassin de compensation d'Espinasses.}

Les trente quatre stations et transects d'échantillonnage sont localisés sur les figures 2 et 3.

Cinq taxons représentent la quasi totalité d'un peuplement peu diversifié : deux potamots (Groenlandia densa et Potamogeton pectinatus) et trois catégories d'algues (des Characées, des cladophores et des spirogyres).

Le substrat vaseux est favorable à tous les végétaux sauf aux cladophores. Les spirogyres se trouvent souvent en épiphytes sur les potamots et sur les Characées.

Les Characées sont peu représentées en faciès lotique.

Les épaisseurs de vase ont été relevées en août 1985 et mai 1986.

Les plantes fixées (Characées et Potamogétonacées), poussent principalement dans les zones envasées, là où le courant est peu rapide et les fonds plus importants ( $\geqslant 40 \mathrm{~cm}$ ).

Dans les zones de rapides et peu profondes, les plantes sont absentes ou chétives, en revanche les Cladophores, fixés sur les gros blocs y abondent.

Enfin les spirogyres montrent un développement important dans les secteurs d'eau dormante : à l'aval immédiat du bassin de compensation (transect 1), au droit de la décharge (station 17) et dans le grand pool à l'amont du pont de Rochebrune (transects 2 à 27).

Les données de biomasse sont présentées synthétiquement dans les tableaux 3 et 4.

Evaluation quantitative de la biomasse.

Compte tenu que seulement quatre transects ont été échantillonnés en septembre et novembre à l'aval du pont de Rochebrune, les données du tableau 4 doivent être considérées comme semi quantitatives.

En novembre les 30 transects et stations échantillonnés jusqu'au pont 
Tableau 3 - Biomasse végétale en poids sec dans les cinq tronçons délimités sur les figures 8, 9, 10 d’après les relevés effectués en août, septembre et novembre 1985.

\begin{tabular}{|c|c|c|c|c|c|c|c|c|c|c|c|c|c|c|}
\hline \multirow{2}{*}{$\begin{array}{c}\text { Tronçon } \\
\text { (transects) }\end{array}$} & \multirow{2}{*}{ Superficie } & \multirow{2}{*}{ Mois } & \multicolumn{2}{|c|}{ G. densa } & \multicolumn{2}{|c|}{ P. pectinatus } & \multicolumn{2}{|c|}{ Characées } & \multicolumn{2}{|c|}{ Cladophora $s p$} & \multicolumn{2}{|c|}{ Spirogyra sp } & \multicolumn{2}{|c|}{ Total } \\
\hline & & & $\mathrm{kg}$ & $\mathrm{kg} / \mathrm{ha}$ & $\mathrm{kg}$ & $\mathrm{kg} / \mathrm{ha}$ & $\mathrm{kg}$ & $\mathrm{kg} / \mathrm{ha}$ & $\mathrm{kg}$ & $\mathrm{kg} / \mathrm{ha}$ & $\mathrm{kg}$ & $\mathrm{kg} / \mathrm{ha}$ & $\mathrm{kg}$ & $\mathrm{kg} / \mathrm{ha}$ \\
\hline \multirow{3}{*}{$\left(\begin{array}{l}1 \\
1 \\
a\end{array}\right.$} & \multirow{3}{*}{$\begin{array}{c}34957 \mathrm{~m}^{2} \\
3,5 \mathrm{ha}\end{array}$} & Août & 2078 & 594 & 45 & 12,8 & 6496 & 1856 & 293 & 84 & 47 & 13 & 8959 & 2560 \\
\hline & & Septembre & 239 & 68 & 78 & 22 & 12731 & 3638 & 44 & 13 & 19 & 5 & 13111 & 3 746 \\
\hline & & Novembre & 452 & 129 & - & - & 8532 & 2438 & 259 & 74 & 67 & 19 & 9310 & 2660 \\
\hline \multirow{3}{*}{$\begin{array}{l}\text { II } \\
(8 \text { a 11) }\end{array}$} & \multirow{3}{*}{$\begin{array}{c}22704 \mathrm{~m}^{2} \\
2,3 \mathrm{ha}\end{array}$} & Aoùt & 1223 & 539 & 2920 & 1270 & 877 & 381 & 28 & 12 & - & - & 5048 & 2195 \\
\hline & & Septembre & 908 & 395 & 1832 & 797 & - & - & 8 & 3,5 & - & - & 2748 & 1195 \\
\hline & & Novembre & 154 & 68 & 253 & 110 & 73 & 32 & 183 & 81 & - & - & 663 & 288 \\
\hline \multirow{3}{*}{$(12$ à 20) } & \multirow{3}{*}{$\begin{array}{c}13173 \mathrm{~m}^{2} \\
1,3 \mathrm{ha}\end{array}$} & Août & 1220 & 939 & 6,6 & 5 & 33 & 25 & 22 & 17 & - & - & 1282 & 986 \\
\hline & & Septembre & 106 & 81 & 0,1 & - & 2997 & 2305 & 80 & 61 & 48 & 36 & 3231 & 2485 \\
\hline & & Novembre & 143 & 110 & 70 & 54 & 1609 & 1237 & 31 & 23,8 & 48 & 36 & 1901 & 1462 \\
\hline \multirow{3}{*}{$\begin{array}{c}\text { IV } \\
(21 \stackrel{a}{a} 27)\end{array}$} & \multirow{3}{*}{$\begin{array}{c}48897 \mathrm{~m}^{2} \\
4,9 \text { ha }\end{array}$} & Août & 2706 & 552 & 86 & 18 & 4715 & 962 & 147 & 30 & 353 & 72 & 8007 & 866 \\
\hline & & Septembre & 865 & 176 & - & - & 8225 & 1679 & - & - & 526 & 107 & 9616 & 435 \\
\hline & & Novembre & - & - & - & - & - & - & - & - & - & - & $\begin{array}{r}3973 \\
\text { (1) }\end{array}$ & $\begin{array}{r}811 \\
(1)\end{array}$ \\
\hline \multirow{3}{*}{$\begin{array}{c}V \\
(28 \mathrm{a} 30)\end{array}$} & \multirow{3}{*}{$\begin{array}{c}4267 \mathrm{~m}^{2} \\
0,4 \mathrm{ha}\end{array}$} & Août & 333 & 832 & 5,5 & 14 & 3 & 8 & 5 & 10 & - & - & 347 & 866 \\
\hline & & Septembre & 33 & 83 & 3 & 7 & 95 & 237 & 37 & 92 & 6 & 15 & 174 & 435 \\
\hline & & Novembre & 23 & 57 & - & - & 21,5 & 54 & 1 & 2 & 0,1 & 0,2 & 46 & 114 \\
\hline
\end{tabular}

(1) En l'absence de relevés, on a admis que ce tronçon représente $25 \%$ de la biomasse totale des tronçons I à V. 
Tableau 4 - Biomasse végétale en poids sec dans les quatre tronçons entre le pont de Rochebrune et le pont de Tallard (figures 11, 12) d'après les relevés de septembre et novembre 1985.

\begin{tabular}{|c|c|c|c|c|c|c|c|c|c|c|c|c|c|c|}
\hline \multirow{2}{*}{$\begin{array}{c}\text { Tronçon } \\
\text { (transects) }\end{array}$} & \multirow{2}{*}{ Superficie } & \multirow{2}{*}{ Mois } & \multicolumn{2}{|c|}{ G. densa } & \multicolumn{2}{|c|}{ P. pectinatus } & \multicolumn{2}{|c|}{ Characées } & \multicolumn{2}{|c|}{ Cladophores } & \multicolumn{2}{|c|}{ Spirogyra } & \multicolumn{2}{|c|}{ Total } \\
\hline & & & $\mathrm{kg}$ & $\mathrm{kg} / \mathrm{ha}$ & $\mathrm{kg}$ & $\mathrm{kg} / \mathrm{ha}$ & $\overline{\mathrm{kg}}$ & $\mathrm{kg} / \mathrm{ha}$ & $\mathrm{kg}$ & $\mathrm{kg} / \mathrm{ha}$ & $\mathrm{kg}$ & $\mathrm{kg} / \mathrm{ha}$ & $\mathrm{kg}$ & $\mathrm{kg} / \mathrm{ha}$ \\
\hline \multirow{2}{*}{$\begin{array}{l}V I \\
31\end{array}$} & \multirow{2}{*}{$\begin{array}{l}113750 \\
11,4 \text { ha }\end{array}$} & Septembre & 1477 & 129,6 & 430 & 37,7 & 4596 & 403,2 & - & - & 180 & 15,8 & 6683 & 586,2 \\
\hline & & Novembre & 753 & 66,1 & - & - & 2990 & 262,3 & 1520 & 133,3 & 196 & 17,2 & 5429 & 478,9 \\
\hline \multirow{2}{*}{$\begin{array}{l}\text { VII } \\
32\end{array}$} & \multirow{2}{*}{$\begin{array}{l}215000 \\
21,5 \text { ha }\end{array}$} & Septembre & 422 & 19,6 & 4221 & 196,3 & - & - & - & - & - & - & 4643 & 216,0 \\
\hline & & Novembre & - & - & 351 & 16,3 & - & - & 398 & 18,5 & - & - & 749 & 34,8 \\
\hline \multirow{2}{*}{$\begin{array}{l}\text { VIII } \\
33\end{array}$} & \multirow{2}{*}{$\begin{array}{l}86250 \\
8,6 \text { ha }\end{array}$} & Septembre & - & - & - & - & 2562 & 297,9 & 22614 & 2629,5 & - & - & 25176 & 2927,4 \\
\hline & & Novembre & - & - & - & - & 5807 & 675,2 & 860 & 100,0 & - & - & 6667 & 775,2 \\
\hline \multirow{2}{*}{$\begin{array}{l}1 \mathrm{XX} \\
34\end{array}$} & \multirow{2}{*}{$\begin{array}{l}38000 \\
38 \text { ha }\end{array}$} & Septembre & - & - & 22849 & 601 & - & - & - & - & - & - & 22849 & 601,2 \\
\hline & & Novembre & - & - & 81074 & 2133 & - & - & - & - & - & - & 81074 & 2133,5 \\
\hline
\end{tabular}


de Rochebrune (tableau 3), confèrent à ces données un caractère quantitatif permettant une interprétation des biomasses en valeur absolue.

La biomasse végétale totale en poids sec varie entre $319 \mathrm{~kg} / \mathrm{ha}$ dans le tronçon $V$ (amont du pont de Rochebrune en novembre 1985) et $3698 \mathrm{~kg} / \mathrm{ha}$ dans le tronçon I (aval du bassin d'Espinasses en septembre 1985).

Variations spatiales de la couverture végétale.

Le secteur le plus riche en végétaux est, aux trois périodes d'échantillonnage, le premier tronçon, situé à l'aval immédiat du pont d'Espinasses. Le grand bassin en amont du pont de Rochebrune (tronçon IV) est le plus pauvre en août; en septembre et novembre, c'est le tronçon V. II semble donc que l'on observe un gradient décroissant de biomasse en $\mathrm{kg} / \mathrm{ha}$ d'Espinasses au pont de Rochebrune.

Variations de composition de la couverture végétale.

Groenlandia densa est l'espèce la plus fréquente dans les échantillonnages réalisés jusqu'au pont de Rochebrune. Elle disparait pratiquement en aval du pont de l'Archidiacre. Les Characées dominent dans le bras gauche en aval du torrent de Trente Pas (stations 5 et 7). Dans le bassin en amont du pont de Rochebrune (stations 22-27) les Characées sont trouvées jusqu'à $2 \mathrm{~m}$ de profondeur; $G$. densa est absent en dessous de
$1,50 \mathrm{~m}$. Potamogeton pectinatus est dominant aux stations 7 à 11 en aval de l'apport d'eaux usées localisé aux stations 6-7. Cette espèce domine en aval de la Luye (station 34) qui reçoit, après traitement, les eaux usées de la ville de Gap et en aval du rejet des eaux usées de Remollon (station 32).

Les Cladophores sont particulièrement abondants en aval du pont de l'Archidiacre (station 31) dans les prélèvements de septembre 1985 .

Variations temporelles de la couverture végétale.

Dans le tronçon du pont d'Espinasses au pont de Rochebrune, la biomasse maximale est observée fin septembre; ce maximum correspond en fait à une forte augmentation de la biomasse des Characées entre août et septembre alors que les potamots, particulièrement $G$. densa, régressent.

En novembre, la surface occupée par les Cladophores tend à augmenter mais tous les autres taxons régressent à l'exception de $P$. pectinatus à l'aval de la Luye.

Ces observations permettent de proposer, pour 1985, l'évolution temporelie suivante :

G. densa : maximum fin août, décroît fortement en fin septembre ($50 \%)$ et en fin novembre $(-90 \%)$.

$P$. pectinatus: même comportement que $G$. densa; on note toutefois qu'il est très abondant en novembre à l'aval de la Luye. 
Characées: maximum fin septembre, régression de $50 \%$ en fin novembre.

Cladophora: maximum en fin novembre.

\subsection{Charge en phosphore de la végétation.}

La teneur en phosphore total dans les échantillons de macrophytes est indiquée dans le tableau 5.

On note dans chaque taxon une forte variabilité d'un échantillon à l'autre pour une même période de mesures. II apparaît toutefois sur les valeurs moyennes des différences interprétables; les potamots sont plus riches en phosphore que les Cladophores et les Characées:
G. densa et $P$. pectinatus : 2,0 à $3,3 \mathrm{mg}$ par gramme en poids sec.

Cladophora sp. : 1,6 à 2,4 mg par gramme en poids sec.

Characées: 1,0 à 1,2 $\mathrm{mg}$ par gramme en poids sec.

Chez les Potamots on observe une augmentation de la teneur en phosphore ainsi qu'une réduction du taux d'humidité entre août et novembre.

Les quantités de phosphore contenues dans la végétation en Durance dans les tronçons I à $V$ du pont d'Espinasses au pont de Rochebrune sont indiquées dans le tableau 6 .

Une évaluation faite sur le bassin d'Espinasses permet d'obtenir le bilan global jusqu'au pont de Rochebrune (tableau 7 ).

Tableau 5. - Teneur en phosphore rapportée au poids sec $(P(\mathrm{mg} / \mathrm{g} P S))$ et rapport du poids Sec au poids frais (E(PS/PF)) dans les échantillons de macrophytes prélevés en août, septembre et novembre 1985 en Durance. On a indiqué entre parenthèses la station où les valeurs minimale (MIN) et maximale (MAX) ont été trouvées.

\begin{tabular}{|c|c|c|c|c|c|c|c|}
\hline & \multicolumn{2}{|c|}{ Août } & \multicolumn{2}{|c|}{ Septembre } & \multicolumn{2}{|c|}{ Novembre } \\
\hline & & $\mathrm{P}(\mathrm{mg} / \mathrm{gPs})$ & $E(P S / P F)$ & $\mathrm{P}(\mathrm{mg} / \mathrm{gPs})$ & $E(P S / P F)$ & $\mathrm{P}(\mathrm{mg} / \mathrm{gPs})$ & $E(P S / P F)$ \\
\hline $\begin{array}{l}\text { Groenlan- } \\
\text { dia densa }\end{array}$ & $\begin{array}{l}\text { MIN } \\
\text { MAX } \\
\text { MOY }\end{array}$ & $\begin{array}{l}1,30(1) \\
4,37(18) \\
2,05\end{array}$ & $\begin{array}{l}0,07 \\
0,11 \\
0,17\end{array}$ & $\begin{array}{l}1,16(33) \\
6,27(7) \\
2,9\end{array}$ & $\begin{array}{l}0,07 \\
0,13 \\
0,19\end{array}$ & $\begin{array}{l}2,23(1) \\
5,62(8) \\
3,33\end{array}$ & $\begin{array}{l}0,06 \\
0,15 \\
0,38\end{array}$ \\
\hline $\begin{array}{l}\text { Potamoge- } \\
\text { ton pecti- } \\
\text { natus }\end{array}$ & $\begin{array}{l}\text { MIN } \\
\text { MAX } \\
\text { MOY }\end{array}$ & $\begin{array}{l}1,13(30) \\
4,28(10) \\
2,29\end{array}$ & $\begin{array}{l}0,10 \\
0,20 \\
0,13\end{array}$ & $\begin{array}{l}1,19(31) \\
3,49(32) \\
2,64\end{array}$ & $\begin{array}{c}0,10 \\
0,20 \\
0,15\end{array}$ & $\begin{array}{c}1,26(33) \\
4,97(10) \\
-\end{array}$ & $\begin{array}{l}0,12 \\
0,24 \\
0,18\end{array}$ \\
\hline $\begin{array}{l}\text { Chara. } \\
\text { sp. }\end{array}$ & $\begin{array}{l}\text { MIN } \\
\text { MAX } \\
\text { MOY }\end{array}$ & $\begin{array}{l}0,71(13) \\
1,31(1) \\
0,95\end{array}$ & $\begin{array}{l}0,16 \\
0,25 \\
0,21\end{array}$ & $\begin{array}{l}0,26(33) \\
3,22(5) \\
1,01\end{array}$ & $\begin{array}{l}0,18 \\
0,23 \\
0,22\end{array}$ & $\begin{array}{l}0,91(2) \\
1,47(30) \\
1,19\end{array}$ & $\begin{array}{l}0,13 \\
0,43 \\
0,22\end{array}$ \\
\hline $\begin{array}{l}\text { Cladopho- } \\
\text { ra sp. }\end{array}$ & $\begin{array}{l}\text { MIN } \\
\text { MAX } \\
\text { MOY }\end{array}$ & $\begin{array}{c}1,86(14) \\
2,36(28) \\
-\end{array}$ & $\begin{array}{l}0,13 \\
0,18 \\
0,15\end{array}$ & $\begin{array}{l}0,46(33) \\
2,12(15) \\
1,59\end{array}$ & $\begin{array}{l}0,12 \\
0,23 \\
0,17\end{array}$ & $\begin{array}{l}1,18(33) \\
3,14(9) \\
2,36\end{array}$ & $\begin{array}{l}0,23 \\
0,41 \\
0,32\end{array}$ \\
\hline $\begin{array}{l}\text { Spirogyra } \\
\text { sp. }\end{array}$ & $\begin{array}{l}\text { MIN } \\
\text { MAX } \\
\text { MOY }\end{array}$ & $\begin{array}{c}0,46(12) \\
1,87(18) \\
-\end{array}$ & $\begin{array}{l}0,08 \\
0,11 \\
0,10\end{array}$ & $\begin{array}{l}\text { une seule } \\
\text { valeur } \\
4,34(12)\end{array}$ & $\begin{array}{c}0,07 \\
-\end{array}$ & 2,09 & 0,08 \\
\hline
\end{tabular}

Pour localiser les stations voir les figures 3 et 4. 
Tableau 6. - Quantité de phosphore (en $\mathrm{kg}$ ) dans la biomasse végétale en Durance du pont d'Espinasses au pont de Rochebrune.

\begin{tabular}{|c|c|c|c|c|c|c|}
\hline $\begin{array}{c}\text { Tronçon } \\
\text { (trans- } \\
\text { ects) }\end{array}$ & Mois & G. densa & P. pectinatus & Characées & $\begin{array}{c}\text { Cladophora } \\
\text { sp. }\end{array}$ & $\begin{array}{c}\text { Spirogyra } \\
\text { sp. }\end{array}$ \\
\hline \multirow{3}{*}{$\left(\begin{array}{l}1 \\
\text { à } 7)\end{array}\right.$} & Août & 2,79 & 0,08 & 5,56 & 0,54 & 0,02 \\
\hline & Septembre & 0,61 & 0,24 & 13,67 & 0,07 & \\
\hline & Novembre & 1,17 & - & 9,52 & 0,28 & \\
\hline \multirow{3}{*}{$\begin{array}{c}\text { II } \\
(8 \text { à 11) }\end{array}$} & Août & 2,01 & 7,63 & 0,28 & 0,05 & \\
\hline & Septembre & 3,47 & 5,77 & - & 0.02 & \\
\hline & Novembre & 0,7 & 1,03 & 0,08 & 0,45 & \\
\hline \multirow{3}{*}{$\begin{array}{c}\text { III } \\
(12 \mathrm{a} \\
20)\end{array}$} & Août & 3,31 & - & 0,02 & 0,04 & \\
\hline & Septembre & 0,25 & - & 2,68 & 0,15 & 0,2 \\
\hline & Novembre & 0,66 & - & 1,67 & 0,06 & \\
\hline \multirow{3}{*}{$\begin{array}{c}\text { IV } \\
(21 \mathrm{a} \\
27)\end{array}$} & Août & 5,03 & 0,10 & 16,69 & 0,21 & 0,16 \\
\hline & Septembre & 4,93 & - & 4,98 & - & 2,24 \\
\hline & Novembre & - & - & - & - & - \\
\hline \multirow{3}{*}{$\begin{array}{c}V \\
(28 \mathrm{a} \\
30)\end{array}$} & Août & 0,58 & 0,02 & - & 0,21 & 0,16 \\
\hline & Septembre & 0,04 & - & 0,07 & - & 2,24 \\
\hline & Novembre & 0,06 & - & 0,09 & - & - \\
\hline
\end{tabular}

Tableau 7. - Quantité de phosphore (en $\mathrm{kg}$ ) contenu dans la végétation macrophytique.

\begin{tabular}{|l|c|c|c|c|c|}
\cline { 2 - 6 } \multicolumn{1}{c|}{} & \multicolumn{2}{c|}{ Bassin d'Espinasses } & \multicolumn{3}{c|}{ Durance jusqu'au pont de Rochebrune } \\
\cline { 2 - 6 } \multicolumn{1}{c|}{} & août & septembre & août & septembre & novembre \\
\hline G. densa & 14,5, & 3,2 & 13,72 & 9,30 & 2,59 \\
\hline P. pectinatus & - & - & 7,83 & 6,01 & 1,29 \\
\hline Characées & 71,7 & 48,4 & 22,55 & 21,45 & 11,36 \\
\hline Cladophora sp. & - & - & 0,84 & 0,29 & 0,79 \\
\hline Total & 86,2 & 51,6 & 44,94 & 37,05 & 16,03 \\
\hline
\end{tabular}




\section{5 - DONNEES DE QUALITE D'EAU.}

\subsection{Données collectées par les stations automatiques.}

Deux stations automatiques équipées de modules MOAMED ont été installées en juin 1985 au pont de Rochebrune et au pont de l'Archidiacre. Les stations fournissent en continu des mesures de température, $\mathrm{pH}$, conductivité et oxygène dissous.

Pendant la période des relevés de biomasse, jusqu'en novembre 1985, les stations permettent de définir les évolutions de qualité d'eau.

Les figures 4 et 5 rapportent les valeurs moyennes mensuelles et les valeurs minimales et maximales enregistrées aux deux stations.

\section{Température.}

La Durance est alimentée par l'eau de fond de la retenue de Serre-Ponçon turbinée au barrage. Cette origine explique les températures basses et relativement constantes relevées dans le bassin de compensation d'Espinasses (environ $10^{\circ} \mathrm{C}$ ). En aval, la température moyenne estivale reste basse à Rochebrune $\left(12^{\circ} \mathrm{C}-13^{\circ} \mathrm{C}\right)$, elle est plus élevée à l'Archidiacre $\left(14^{\circ} \mathrm{C}-17^{\circ} \mathrm{C}\right)$.

Les variations nycthémérales de température sont élevées. L'amplitude thermique journalière atteint environ $4^{\circ} \mathrm{C}$ à Rochebrune et environ $6{ }^{\circ} \mathrm{C}$ au pont de l'Archidiacre (tableau 8).

$p H$

Le pH est alcalin avec des moyennes mensuelles entre 8,09 et 8,35 au pont de Rochebrune et entre 7,89 et 8,24 au pont de l'Archidiacre.

Tableau 8. - Amplitude thermique journalière de juin à novembre 1985; valeurs maximales et moyennes mensuelles.

\begin{tabular}{|r|c|c|c|c|c|c|}
\hline Station & juin & juillet & août & septembre & octobre & novembre \\
\hline $\begin{array}{r}\text { max. } \\
\text { Rochebrune }\end{array}$ & 4,5 & 4,6 & 4,7 & 3,6 & 2,7 & 1,9 \\
moy. & 4,4 & 4,5 & 3,6 & 2,9 & 2,3 & 1 \\
\hline $\begin{array}{r}\text { max. } \\
\text { Archidiacre }\end{array}$ & 7,9 & 7,9 & 7,9 & 6,9 & 5,1 & 3,7 \\
moy. & 5,7 & 6 & 6 & 5,4 & 3,7 & 2,3 \\
\hline
\end{tabular}

Tableau 9. - Moyennes mensuelles des fluctuations journalières de pH de juin à novembre 1985.

\begin{tabular}{|l|c|c|c|c|c|c|}
\hline \multicolumn{1}{|c|}{ Station } & Juin & juillet & août & septembre & octobre & novembre \\
\hline Rochebrune & 0,48 & - & 0,52 & 0,58 & 0,54 & 0,23 \\
\hline Archidiacre & 0,30 & - & 0,37 & 0,45 & 0,40 & 0,27 \\
\hline
\end{tabular}



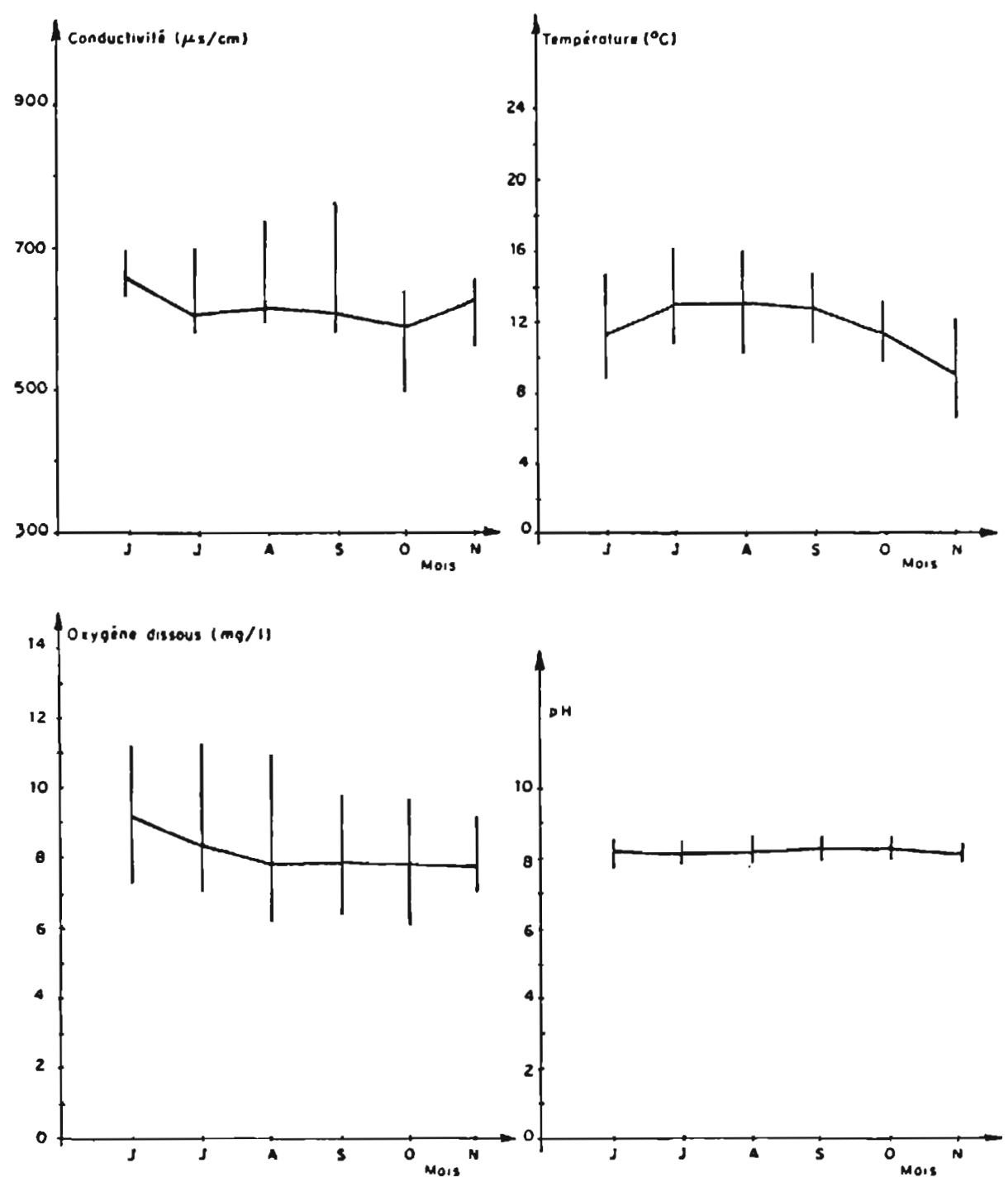

Fig. 4. - Evolution des paramètres de qualité d'eau mesurés au pont de Rochebrune de juin à novembre 1985.

Les valeurs moyennes mensuelles sont entourees de lintervalle de variation (valeurs minimum et maximum). 

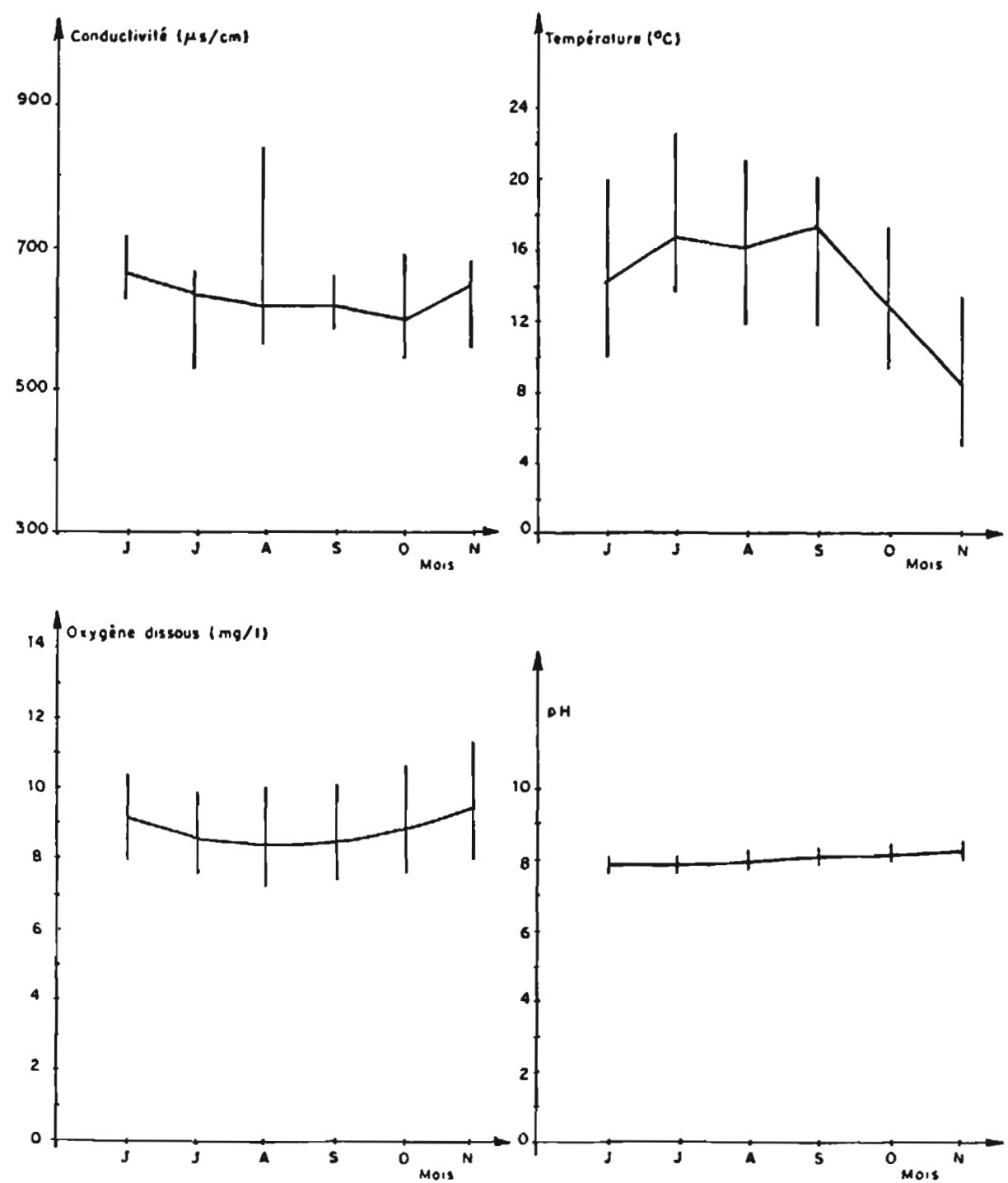

Fig. 5 - Evolution des paramètres de qualité d'eau mesurés au pont de l'Archidiacre de juin à novembre 1985.

Les valeurs moyennes mensuelles sont entourées de lintervalle de variation (valeurs minimum ot maximum). 
Comme pour les températures, des variations nycthémérales sont observées, le minimum diurne et le maximum nocturne révèlent l'origine photosynthétique de ces fluctuations périodiques.

Le tableau 9 rapporte les amplitudes journalières de $\mathrm{pH}$, elles sont en moyenne plus élevées à Rochebrune qu'à l'Archidiacre et sont maximum en septembre 1985.

\section{Conductivité.}

Les valeurs relevées au pont de Rochebrune sont relativement stables (environ $320 \mu \mathrm{s} / \mathrm{cm}$ ). Elles correspondent à une assez forte minéralisation. Au pont de l'Archidiacre la conductivité double avec une moyenne de $630 \mu \mathrm{s} / \mathrm{cm}$. L'augmentation de minéralisation peut avoir plusieurs origines: passage de l'eau dans une gravière en exploitation, apports par les torrents et des écoulements diffus.

Le rôle des apports intermédiaires entre les deux stations de mesures est vraisemblablement déterminant dans la croissance de la minéralisation de l'amont vers l'aval. On note sur les restitutions graphiques les
7 juillet, 6 et 25 août 1985, que les pluies d'orages ont provoqué une variation brutale de conductivité qui se manifeste par une augmentation des valeurs.

Les épisodes orageux s'accompagnent d'autre part d'une atténuation des variations nycthémérales d'oxygène dissous et de $\mathrm{pH}$, c'est-à-dire d'une inhibition de la photosynthèse végétale en raison de l'accroissement de la turbidité de l'eau et de la réduction du rayonnement solaire.

\section{Oxygène dissous.}

Au pont de Rochebrune, les moyennes mensuelles diminuent de juin à août $(9,3 \mathrm{mg} / \mathrm{l}$ en juin, $7,8 \mathrm{mg} / \mathrm{l}$ en août) puis elles restent stables d'août à novembre. A l'Archidiacre, les moyennes mensuelles sont plus élevées (9,6 $\mathrm{mg} / \mathrm{l}$ en novembre, $8,5 \mathrm{mg} / \mathrm{l}$ en août); elles diminuent également de juin à août.

Les variations nycthémérales d'oxygène dissous ont le même comportement que celles du $\mathrm{pH}$ : elles sont plus élevées à Rochebrune qu'à l'Archidiacre et sont maximum en septembre (tableau 10). Ce tableau rapporte également les teneurs mini-

Tableau 10. - Amplitude journalière moyenne mensuelle en oxygène dissous ( $A$ en $\mathrm{mg} / \mathrm{l}$ ) et minimum relevé ( $\mathrm{m}$ en $\mathrm{mg} / \mathrm{l}$ ).

* capteurs en panne à partir du 17/07/85.

* capteurs en panne à partir du 16/11/85.

\begin{tabular}{|c|c|c|c|c|c|c|c|}
\hline Station & & juin & juillet & août & septembre & octobre & novembre \\
\hline Rochebrune & A & 2,7 & $*-$ & 2,5 & 3,0 & 2,9 & $1,3 \cdots$ \\
& m & 7,6 & $* 7,0$ & 6,1 & 6,3 & 6,0 & $7,1 *$ \\
\hline Archidiacre & A & 1,5 & $\bullet-$ & 2,0 & 2,4 & 2,1 & 1,7 \\
& m & 8,0 & 7,7 & 7,3 & 7,5 & 7,7 & 8,1 \\
\hline
\end{tabular}


Tableau 11. - Taux de saturation en oxygène dissous en \%; moyennes mensuelles, valeurs minimum et maximum aux deux stations de mesures de juin à novembre 1985 .

\begin{tabular}{|c|c|c|c|c|c|c|c|}
\hline Station & & juin & juillet & août & septembre & octobre & novembre \\
\hline \multirow{3}{*}{ Rochebrune } & $\min$ & 68 & 63 & 57 & 60 & 54 & 61 \\
& $\operatorname{moy}$ & $(84)$ & $(78)^{*}$ & $(74)$ & $(73)$ & $(70)$ & $(66)^{*}$ \\
& $\max$ & 111 & 110 & 107 & 92 & 87 & 82 \\
\hline \multirow{3}{*}{ Archidiacre } & $\min$ & 82 & 83 & 76 & 76 & 76 & 75 \\
& $\operatorname{moy}$ & $(90)$ & $(88)^{*}$ & $(86)$ & $(85)$ & $(84)$ & $(82)$ \\
& $\max$ & 97 & 87 & 107 & 104 & 98 & 94 \\
\hline
\end{tabular}

- (moyenne de 2 semaines).

mum en oxygène dissous relevées aux deux stations.

A Rochebrune, pendant la période de mesures, l'oxygène dissous est resté supérieur ou égal à $6 \mathrm{mg} / \mathrm{l}$ et au-dessus de $7,3 \mathrm{mg} / \mathrm{l}$ à l'Archidiacre.

Les taux de saturation calculés à partir des valeurs moyennes mensuelles de température et d'oxygène dissous sont compris entre $66 \%$ et $84 \%$ à Rochebrune et entre $82 \%$ et $90 \%$ à l'Archidiacre (tableau 11).

Des mesures d'oxygène dissous par la méthode chimique (Winkler) ont été réalisées à la fin du mois d'août à $06 \mathrm{~h} 00$ et $19 \mathrm{~h} 00(\mathrm{TU}+2)$ en 11 stations situées entre les ponts d'Espinasses et de Rochebrune (figure 6). A $6 \mathrm{~h} \mathrm{00,} \mathrm{au} \mathrm{moment} \mathrm{du}$ lever du soleil, l'oxygène dissous est proche du minimum nocturne. La plus faible valeur relevée était $6,9 \mathrm{mg} / \mathrm{l}$ à la station 23 sous la végétation en rive gauche.

\subsection{Nutriments.}

Des prélèvements d'eau de surface ont été réalisés lors des campagnes de mesures. Les échantillons ont été congelés sur place à l'usine de SerrePonçon puis acheminés au laboratoire d'Hydrobiologie de l'Université de Provence pour analyses d'azote et de phosphore.

\section{Bassin de Compensation d'Espi- nasses.}

Les teneurs en azote des nitrates restent stables dans le bassin avec une valeur d'environ $0,2 \mathrm{mg} / \mathrm{l}$; l'azote total est également stable avec environ $0,3 \mathrm{mg} / \mathrm{l}$. L'ensemble du bassin est sous la dépendance des apports d'eau de fond de Serre-Ponçon.

L'épisode orageux du 25/08/85 a produit une augmentation d'azote dans l'eau de la Blanche, affluent en rive gauche proche de l'entrée du canal de Curbans; l'on y a dosé $0,4 \mathrm{mg} / \mathrm{l}$ de $\mathrm{N}-\mathrm{NO}_{3}$ et $0,6 \mathrm{mg} / \mathrm{l}$ de $\mathrm{N}$ TOTAL le 26 août.

Les concentrations en phosphates et phosphore total sont également relativement stables $(6-20 \mu \mathrm{g} / \mathrm{l}$ en P$\mathrm{PO}_{4}$, et 20 à $48 \mu \mathrm{g} / \mathrm{l}$ en $\mathrm{P}$ TOTAL). En novembre on observe une nette réduction de la teneur en phos- 

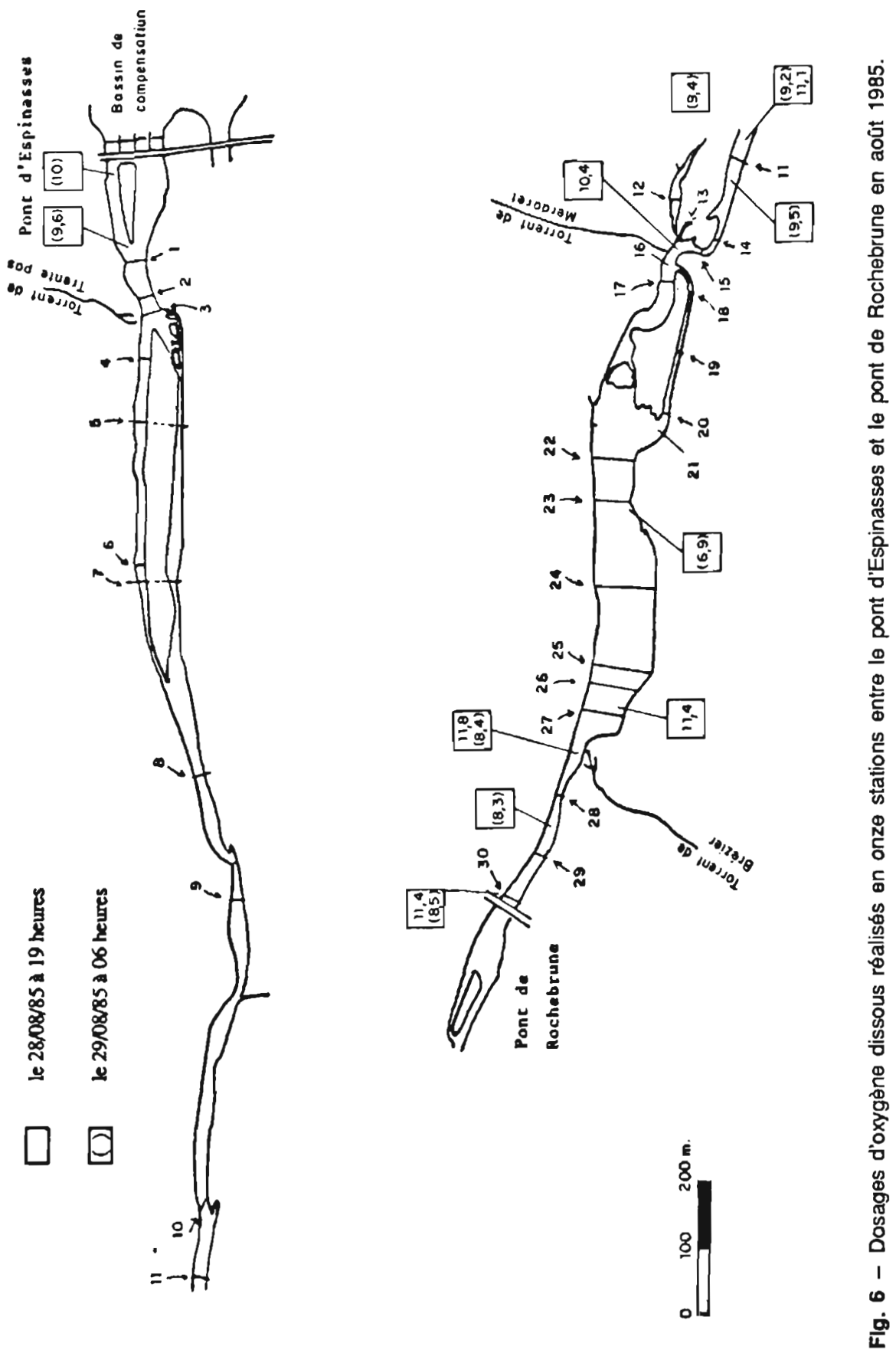
Tableau 12. - Concentrations en $N$ et $P$ du transect 24, milieu du pool.

\begin{tabular}{|l|c|c|c|c|}
\cline { 2 - 5 } \multicolumn{1}{c|}{} & \multicolumn{2}{c|}{ septembre } & \multicolumn{2}{c|}{ novembre } \\
\cline { 2 - 5 } \multicolumn{1}{c|}{} & Surface & Fond $(3 \mathrm{~m})$ & Surface & Fond $(3 \mathrm{~m})$ \\
\hline $\mathrm{P}-\mathrm{PO}_{4}(\mu \mathrm{g} /)$ & 12 & 7 & 18 & 2 \\
\hline $\mathrm{P}$ TOTAL $(\mu \mathrm{g} /)$ & 29 & 160 & 42 & 23 \\
\hline $\mathrm{N}-\mathrm{NO}_{3}(\mu \mathrm{g} /)$ & 270 & 209 & 257 & 250 \\
\hline $\mathrm{N}$ TOTAL $(\mu \mathrm{g} /)$ & 305 & 578 & 307 & 295 \\
\hline
\end{tabular}

phates : 3-4 $\mu \mathrm{g} /$ en sortie de SerrePonçon, et $2 \mu \mathrm{g} / \mathrm{l}$ à l'extrémité aval du bassin.

\section{La Durance en aval du bassin.}

Sur cette partie du cours de la Durance, de nombreux apports latéraux (torrents et égouts) sont une source d'azote et de phosphore. Les eaux usées d'Espinasses, de Rémollon et surtout de Gap, qui transitent par la Luye, sont une source non négligeable de nutriments.

On observe en aval de ces apports une augmentation transitoire des concentrations. Si l'on ne tient pas compte de ces points singuliers, la teneur en phosphore des ortophosphates et la concentration en azote nitrique s'établissent autour des valeurs suivantes:

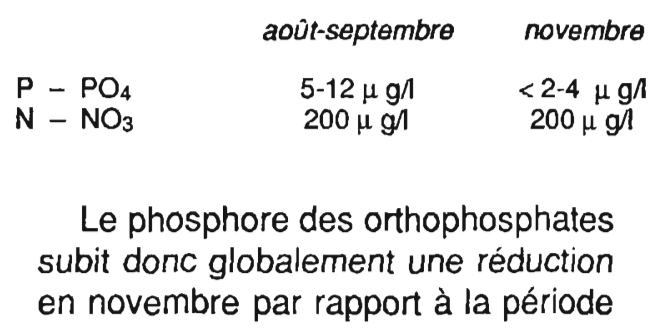

estivale. Pour le phosphore comme pour l'azote, les concentrations décroissent à l'aval des apports ponctuels, sans doute du fait de la consommation par les végétaux.

Dans le pool situé en amont du pont de Rochebrune (transect 24), la concentration en phosphore total est plus élevée près du fond qu'en surface dans les prélèvements de septembre. L'azote et le phosphore organiques sont générés par la dégradation des herbiers à Potamogetonacées à la fin septembre.

\subsection{Matières en suspension.}

Le 17 juillet, les dosages de matières en suspension totales effectués en Durance donnent des valeurs comprises entre 3 et $30 \mathrm{mg} / \mathrm{l}$ :

\section{Station}

MEST (mg/)

1 (débit réservé à Espinasses)

10,48

2

3,37

30,43

30

6,80

32

23,20

Pont de l'Archidiacre 
Tableau 13. - Matières en suspension totales (MEST) dosées sur les prélèvements effectués les 25 et 26 août sur le tronçon étudié (I) et en Durance à Valernes et dans d'autres rivières (II).

\begin{tabular}{|c|c|c|}
\hline Station & MEST le $25 / 08$ (mg/l) & MEST le $26 / 08$ (mg/) \\
\hline $\begin{array}{l}\text { La Blanche } \\
\text { Bassin d'Espinasses } \\
\text { Pont de Rochebrune (30) } \\
\text { Torrent de Trente pas } \\
\text { Torrent de la Clapouse } \\
\text { Torrent de Théus }\end{array}$ & $\begin{array}{l}19 \text { h } 00: 10838,6 \\
19 \text { h } 00: 3681,7 \\
19 \text { h } 00: 9000,0\end{array}$ & $\begin{array}{l}15 \text { h } 30: 350,0 \\
15,7 \\
17 \text { h } 15: 10,9 \\
8 \text { h } 40: 84,2\end{array}$ \\
\hline $\begin{array}{l}\text { Il } \\
\text { Durance à Valernes } \\
\text { Sasse à Valernes } \\
\text { Buëch à Ribiers }\end{array}$ & & $\begin{array}{lll}11 & \text { h } 20: & 1886,5 \\
11 & \text { h } 05: & 157,1 \\
10 & \text { h } 30: & 324,8\end{array}$ \\
\hline
\end{tabular}

A la fin août l'épisode orageux du 25 a permis de mettre en évidence les apports latéraux par les torrents les 25 et 26 août (tableau 13).

Les torrents de la rive droite apportent le 25 août une quantité élevée de matières en suspension: environ $10 \mathrm{~g} / \mathrm{l}$.

\section{6 - DISCUSSION.}

Les données collectées au cours de l'été et de l'automne fournissent les principales caractéristiques physiques et chimiques de leur habitat, l'écosystème durancien à l'aval de Serre-Ponçon. Nous avons d'autre part évalué l'importance de la biomasse végétale en poids frais et poids sec ainsi que la quantité de phosphore contenu dans la végétation. Dans ce chapitre nous proposons de comparer ces résultats à ceux publiés dans la littérature.

\subsection{Ecologie des macrophytes.}

Les Characées, groupe dominant qui représentait en septembre 1985 environ $80 \%$ de la biomasse totale, sont abondants dans les zones profondes: le bassin de compensation d'Espinasses (sous 4 à $5 \mathrm{~m}$ d'eau), le pool en amont du pont de Rochebrune. La présence des Chara dans les eaux turbides et/ou à des profondeurs importantes s'explique selon Dale (1986) par une adaptation aux faibles éclairements. Le maximum de développement des Characées, d'après nos relevés, se situe à la fin septembre; ce fait confirme la poursuite de la croissance au-delà de la période du maximum de rayonnement solaire. II faut toutefois remarquer que les Charas sont également abondants dans des secteurs peu profonds à l'aval du bassin de compensation d'Espinasses. De même, dans le bras gauche, en aval du Torrent de Trente Pas, ce groupe forme de vaste tapis excluant toute autre espèce végétale. 
Dans ces "zones à Chara", peu profondes, on note des vitesses d'écoulement faibles, la présence de sédiments fins et peu remaniés. Le substrat vaseux stable serait, selon Corillon (1957) et Pereira Ramos (1983), un facteur essentiel du développement des Chara. Par ailleurs les concentrations en orthophosphates sont faibles : $\leq 10 \mu \mathrm{g} / \mathrm{l}$ en P-PO4. La sensibilité des Chara aux fortes charges en phosphore fait l'objet d'une controverse.

Forsberg (1964) observe des concentrations très faibles en phosphates (parfois $1 \mu \mathrm{g} / \mathrm{l}$ ) dans l'eau des lacs à Chara. Les expériences qu'il réalisa en laboratoire sur Chara globuralis, montrèrent que l'addition d'orthophosphate a un effet inhibiteur sur la croissance. Cette inhibition serait de $70 \%$ pour une concentration de $8 \mu \mathrm{g} / \mathrm{l}$ et totale à $30 \mu \mathrm{g} / \mathrm{l}$ de $\mathrm{P}$ $\mathrm{PO}_{4}$. Dans cette expérience il avait été observé qu'un courant suffisant pour assurer le renouvellement d'eau autour des pieds augmente la capacité de résistance des plants.

Les conclusions de Forsberg ont été contredites par des expérimentations plus récentes qui ont montré l'absence d'effet des concentrations élevées en phosphore minéral 11 à $2 \mathrm{mg} / \mathrm{l})$ sur le taux de croissance de plusieurs espèces de Chara (Blindow, 1988). L'absence de Characées dans les lacs eutrophisés s'expliquerait plutôt, selon d'autres auteurs, par l'accroissement de l'extinction de la lumière liée à la forte biomasse phytoplanctonique que par la teneur en phosphore.
Les Characées sont également sensibles aux fortes teneurs en matières organiques dissoutes (Pip, 1984) et n'occupent que les sites très peu ou non pollués par des rejets d'eaux usées (Ozimek, 1978). Lachavanne, (1985), les cite parmi les espèces les plus communes des lacs oligotrophes et mésotrophes (tableau 13).

Potamogeton pectinatus a été trouvé en quantités considérables dans les sites pollués par les rejets d'eau usées: aval des égouts d'Espinasses, de Rémollon et de Gap. De nombreux auteurs (Sculthorpe 1967, Haslam 1975, Ozimek 1978, Ho 1979, Dykyjova et al., 1985, La Chavanne et al., 1986) ont constaté la présence de $P$. pectinatus à proximité d'effluents d'origine organique. Cette espèce est capable de supporter de fortes concentrations en ammoniaque (Dykyjova et al., 1985) et en matières organiques dissoutes (Pip 1984). La Chavanne (1985), le place parmi les végétaux les plus communs dans les lacs eutrophes (tableau 14). Enfin, la forme linéaire de ses feuilles qui réduit le dépôt de particules solides et le développement des épiphytes constitue un avantage sur d'autres espèces (Sculthorpe, 1967).

Toutes ces caractéristiques en font des plantes particulièrement bien adaptées à la colonisation des secteurs les plus pollués, capables même de proliférer à faible distance des rejets ciomestiques (Ozimek, 1978).

Or, dans la Durance, on constate que, si la présence des $P$. pectinatus 
Tableau 14. - Typologie trophique des macrophytes des lacs suisses selon Lachavanne (1985).

\begin{tabular}{|c|c|c|}
\hline Etat trophique & \multicolumn{2}{|c|}{ Espèces les plus communes } \\
\hline Oligotrophe & $\begin{array}{l}\text { Nitella sp } \\
\text { Chara sp. } \\
\text { Ranunculus sp. } \\
\text { Potamogeton graminaeus } \\
\text { Potamogeton praelongus } \\
\text { Potamogeton alpinus }\end{array}$ & Lacs de montagne \\
\hline Mesotrophes & $\begin{array}{l}\text { Chara sp. (jusqu'à } 15 \mathrm{~m} \text { ) } \\
\text { Potamogeton perfoliatus } \\
\text { Potamogeton pectinatus } \\
\text { Potamogeton filiformis } \\
\text { Potamogeton graminaeus } \\
\text { Potamogeton pusillus } \\
\text { Potamogeton lucens }\end{array}$ & $\begin{array}{l}\text { Zone littorale } \\
\text { Phragmites australis } \\
\text { Scirpus lacustris } \\
\text { Nymphaea alba }\end{array}$ \\
\hline Eutrophes & $\begin{array}{l}\text { Végétation limitée à } 5-6 \text { m de profondeur } \\
\text { Potamogeton pectinatus } \\
\text { Potamogeton perfoliatus } \\
\text { Potamogeton crispus } \\
\text { Potamogeton lucens } \\
\text { Zannichellia palustris } \\
\text { Myriophyllum spicatum } \\
\text { Ceratophyllum demersum } \\
\text { Nittellopsis oblusa }\end{array}$ & $\begin{array}{l}\text { Zone littorale } \\
\text { Pragmites australis } \\
\text { Typha sp. } \\
\text { Nuphar luteum }\end{array}$ \\
\hline Hypereutrophes & $\begin{array}{l}\text { Végétation limitée à } 2 m \text { de profondeur } \\
\text { Najas marina } \\
\text { Potamogeton crispus. }\end{array}$ & $\begin{array}{l}\text { Zone littorale } \\
\text { Phragmites australis } \\
\text { Nuphar luteum }\end{array}$ \\
\hline
\end{tabular}

est effectivement liée aux arrivées d'eaux usées, leur prolifération n'intervient qu'à environ $200 \mathrm{~m}$ à l'aval des égouts d'Espinasses et à $1000 \mathrm{~m}$ à l'aval de l'embouchure de la Luye (eaux usées de Gap).

Cette absence de colonisation peut être la conséquence d'un substrat impropre, limitant la production. D'après les données de la littérature $P$. Pectinatus semble exigeant en ce qui concerne la texture du substrat.

Anderson (1978) et Ho (1979) constatèrent que les $P$. pectinatus poussant sur les substrats sableux présentaient une biomasse en poids frais beaucoup plus importante que ceux poussant sur les substrats vaseux. Ils sont cependant capables de s'adapter à un substrat argilo-vaseux en l'absence d'une turbulence excessive.

II est probable qu'en Durance la texture du substrat puisse intervenir dans la répartition de $P$. pectinatus.

II est d'autre part possible que la présence de substances toxiques dans les effluents domestiques ou la formation de vase putride, conséquence de l'enrichissement en ma- 
tières organiques du substrat, élimine les macrophytes enracinés dans la zone la plus proche des rejets (Lachavanne, 1985).

Une faible luminosité peut enfin limiter l'extension de cette espèce. Les herbiers de $P$. pectinatus se situent en effet rarement au-dessous de $0,5 \mathrm{~m}$ de profondeur. Anderson (1978), a observé une diminution linéaire de la biomasse de $P$. pectinatus en dessous de 0,6 $\mathrm{m}$ avec une absence totale au-delà de $1 \mathrm{~m}$ de profondeur.

En aval des égouts de Gap, les apports de la Luye doivent augmenter la turbidité de l'eau et concourir, avec la présence d'arbres sur les berges, à réduire l'éclairement solaire. Comme l'espèce $G$. densa, $P$. pectinatus présente son maximum de développement fin août.

Groenlandia densa, contrairement aux Chara et $P$. pectinatus qui montrent une répartition spatiale relativement stricte, est présent dans le bassin d'Espinasses comme dans la rivière. Cette espèce forme des herbiers monospécifiques mais elle est aussi mêlée aux Chara et $P$. pectinatus. L'abondance relative de $G$. densa entre le pont d'Espinasses et le pont de Rochebrune est quasiment constante (environ $600 \mathrm{~kg} / \mathrm{ha}$ ) quel que soit le secteur de prélèvement ou le degré de pollution.

Moins exigeant vis-à-vis de la lumière que l'espèce pectinatus, $G$. densa colonise le bassin de compensation d'Espinasses, jusqu'à une profondeur de 2,50 m. Dans le grand pool en amont du pont de Ro- chebrune, lors de leur extension maximale, en août, ils se montrent capables de concurrencer les Chara pour la colonisation des fonds. Cependant, moins bien adaptés que ces derniers aux faibles luminosités, les $G$. densa sont souvent exclus en septembre des secteurs les plus profonds du pool.

D'autre part, $G$. densa est tolérant vis-à-vis des fortes teneurs en phosphore; Kohler (1971) les récolte dans des eaux présentant des teneurs en phosphates allant de l'état de traces à $0,46 \mathrm{mg} / \mathrm{l}$ et Mattes et Krebs (1974) déterminent expérimentalement leur limite de tolérance à $1 \mathrm{mg} / \mathrm{l}$ de $\mathrm{PO}^{3}$.

Cette espèce supporte de fortes teneurs en nitrates $(5,3 \mathrm{mg} / \mathrm{l})$ et en ammoniaque ( $5 \mathrm{mg} / \mathrm{l})$.

Groenlandia densa préfère les eaux bien minéralisées riches en calcium et se trouve selon Mattes et Krebs (1974), dans une gamme de conductivité de $900 \mu \mathrm{s}$ en moyenne.

Cette tolérance assez large explique les valeurs de biomasses relativement constantes observées jusqu'au pont de Rochebrune.

Les Cladophores se rencontrent principalement dans les zones courantes et peu profondes. lis sont fixés sur les gros blocs, leurs filaments orientés dans le sens du courant. Ces algues sont cependant très sensibles aux crues qui provoquent une diminution de la biomasse par l'arrachage d'un bon nombre de plants (Valet 1959, Freeman 1986). 
Tableau 15. - Biomasse végétale dans le bassin de compensation d'Espinasses et quantité de phosphore dans la végétation.

\begin{tabular}{|c|c|c|c|c|c|c|c|c|}
\hline & \multicolumn{4}{|c|}{ Biomasse } & \multicolumn{4}{|c|}{ Phosphore } \\
\hline & \multicolumn{2}{|c|}{ Masse en $\mathrm{kg}$ de poids sec } & \multicolumn{2}{|c|}{ Masse en $\mathrm{kg} / \mathrm{ha}$ (poids sec) } & \multicolumn{2}{|c|}{ Masse en $\mathbf{k g}$} & \multicolumn{2}{|c|}{ Masse en $\mathrm{kg} / \mathrm{ha}$} \\
\hline & Août & Septembre & Août & Septembre & Août & Septembre & Août & Septembre \\
\hline G. densa & 7000 & 1812 & 62 & 16 & 14,5 & 3,2 & 0,13 & 0,03 \\
\hline Characées & 62923 & 93152 & 560 & 828 & 71,7 & 48,4 & 0,64 & 0,43 \\
\hline Total & 70000 & 95000 & 622 & 844 & 86,00 & 52,00 & 0,77 & 0,46 \\
\hline
\end{tabular}

Tableau 16. - Biomasse végétale en Durance du pont d'Espinasses au pont de Rochebrune et quantité de phosphore dans la végétation.

\begin{tabular}{|c|c|c|c|c|c|c|c|c|c|c|c|c|}
\hline & \multicolumn{6}{|c|}{ Biomasse } & \multicolumn{6}{|c|}{ Phosphore } \\
\hline & \multicolumn{3}{|c|}{ Masse en $\mathrm{kg}$ de poids sec } & \multicolumn{3}{|c|}{ Masse en $\mathrm{kg} / \mathrm{ha}$ (poids sec) } & \multicolumn{3}{|c|}{ Masse en $\mathrm{kg}$} & \multicolumn{3}{|c|}{ Masse en $\mathrm{kg} / \mathrm{ha}$} \\
\hline & $\begin{array}{l}\text { Août } \\
(26-29)\end{array}$ & $\begin{array}{c}\text { Septembre } \\
(23-27)\end{array}$ & $\begin{array}{c}\text { Novembre } \\
(25-28)\end{array}$ & $\begin{array}{c}\text { Août } \\
(26-29)\end{array}$ & $\begin{array}{c}\text { Septembre } \\
(23-27)\end{array}$ & $\begin{array}{c}\text { Novembre } \\
(25-28)\end{array}$ & Août & Septembre & Novembre & Août & Septembre & Novembre \\
\hline G. densa & 7560 & 2150 & 772 & 610 & 173 & 62 & 13,72 & 9,30 & 2,59 & 1,11 & 0,75 & 0,21 \\
\hline P. pectinatus & 3062 & 1913 & 323 & 247 & 154 & 26 & 7,83 & 6,01 & 1,29 & 0,63 & 0,49 & 0,10 \\
\hline Characées & 12124 & 24048 & 10236 & 978 & 1939 & 825 & 22,55 & 21,45 & 11,36 & 0,07 & 0,02 & 0,06 \\
\hline $\begin{array}{l}\text { Cladophora sp. } \\
\text { Spirogyra sp }\end{array}$ & 896 & 768 & 589 & 72 & 62 & 48 & 0,84 & 0,29 & 0,79 & 0,07 & 0,02 & 0,06 \\
\hline . Total & 23642 & 28879 & 11920 & 1907 & 2329 & 961 & 45 & 37 & 16 & 3,63 & 2,99 & 1,29 \\
\hline
\end{tabular}


Les Cladophores sont trouvées dans un large intervalle de concentrations en nutriments. En Durance, nous avons observé un développement des Cladophores en novembre, aux transects 8-11, à l'aval des égouts d'Espinasses où la biomasse passe de 3 à $79 \mathrm{~kg} / \mathrm{ha}$; dans le même temps $P$. pectinatus régressait de 950 à $77 \mathrm{~kg} / \mathrm{ha}$. La succession PotamotsCladophores serait liée au cycle thermique selon Wong et al. (1978).

Les Spirogyres se développent en épiphytes sur les Charas et Potamots dans le bassin de compensation, le pool en amont du pont de Rochebrune et le petit pool en amont du cône de déjection du Torrent de Merdaret (transect 12). On les trouve également sur le fond de vase du bassin de compensation ainsi que dérivant en pleine eau. Les Spirogyres sont bien adaptées à une faible luminosité, puisqu'elies se trouvent jusqu'à $7 \mathrm{~m}$ de profondeur dans le bassin de compensation. En revanche elles ne se trouvent que dérivantes dans les zones courantes; cette espèce se développe sélectivement dans les eaux peu courantes et stagnantes.

\subsection{Biomasse végétale.}

Les tableaux 15 et 16 rapportent les biomasses estimées dans le bassin de compensation et en Durance.

Selon Haslam 1978, les biomasses maximales en poids de matières organiques pour les macrophytes submergés sont comprises entre 0,4 et $0,7 \mathrm{~kg} / \mathrm{m}^{2}$ soit 4000 à $7000 \mathrm{~kg} / \mathrm{ha}$.
Dawson (comm. personnelle) a mesuré des biomasses maximales de 310 à $400 \mathrm{~g} / \mathrm{m}^{2}$ en poids sec soit 3000 à $4000 \mathrm{~kg} / \mathrm{ha}$ sur l'espèce $R a$ nunculus calcareus.

Dans une rivière d'Angleterre (Yare), la biomasse maximum des Potamots atteignait $380 \mathrm{~g} / \mathrm{m}^{2}$ en poids sec soit $3800 \mathrm{~kg} / \mathrm{ha}$ (in Jorgensen, 1979).

En Durance, entre le pont d'Espinasses et le pont de Rochebrune nous avons estimé la biomasse maximum en poids sec à $1940 \mathrm{~kg} / \mathrm{ha}$ pour les Characées et $2330 \mathrm{~kg} / \mathrm{ha}$ pour l'ensemble des macrophytes à la fin du moins de septembre. Ce secteur de Durance figure ainsi parmi les rivières à forte biomasse de macrophytes.

Le maximum de biomasse est atteint en fin septembre en raison de l'importance considérable des Characées à cette période ( $86 \%$ du total). Les Potamots présentent en revanche un maximum en fin août; entre fin août et fin novembre la biomasse de Potamots régresse de $90 \%$.

Le rapport poids sec/poids frais varie entre $13,7 \%$ et $15,6 \%$ chez les Potamots (in Jorgensen, 1979); nous avons obtenu une moyenne de $11 \%$ pour $G$. densa et $13 \%$ pour $P$. pectinatus.

\subsection{Relations entre la végétation et le phosphore.}

D'après nos analyses, $P$. pectinatus présente des teneurs en phosphore total allant de $1,13 \mathrm{mg} / \mathrm{g}$ à $4,97 \mathrm{mg} / \mathrm{g}$ de poids sec. Ces valeurs 
sont inférieures à celles relevées dans des eaux fortement polluées:

- 6,5 mg/g de poids sec dans le Loch Forfar en Ecosse (Ho, 1979),

- 8,2 mg/g de poids sec dans le "Golden Canal» en Tchécoslovaquie (Dykyjova et al. 1985).

Des variations saisonnières sont rapportées dans la littérature.

Vermaak et al. (1982) observent un minimum de $5,91 \mathrm{mg} / \mathrm{g}$ de poids $\mathrm{sec}$ en été et un maximum de $8,6 \mathrm{mg} / \mathrm{g}$ en automne. Les teneurs que nous avons obtenues sur $P$. densus vont dans le même sens avec un maximum en fin novembre.

Dans trois tronçons d'une rivière de Pennsylvanie, Wright et McDonnell (1986), ont trouvé chez $P$. crispus et $P$. foliosus des teneurs en phosphore variant entre $1,2 \mathrm{mg} / \mathrm{g}$ et plus de $8 \mathrm{mg} / \mathrm{g}$ de poids sec. La charge en phosphore tissulaire était la plus faible dans le tronçon où l'eau était la plus pauvre en phosphore total $(17 \mu \mathrm{g} / \mathrm{l})$ et la plus forte dans celui où la teneur atteignait une valeur très élevée (1 $340 \mu \mathrm{g} / \mathrm{l})$ :
Pereira-Ramos (1981) observe des valeurs de $1 \mathrm{mg} / \mathrm{g}$ à $1,7 \mathrm{mg} / \mathrm{g}$ de poids $\mathrm{sec}$, proches des teneurs que nous avons dosées en Durance : $0,5 \mathrm{mg} / \mathrm{g}$ à $1,14 \mathrm{mg} / \mathrm{g}$ dans le bassin de compensation, 0,71 à $1,31 \mathrm{mg} / \mathrm{g}$ dans la rivière en août et $0,91 \mathrm{mg} / \mathrm{g}$ à $1,47 \mathrm{mg} / \mathrm{g}$ en novembre.

Les Cladophores ont des teneurs moyennes de $1,6 \mathrm{mg} / \mathrm{g}$ de poids $\mathrm{sec}$ en septembre et $2,4 \mathrm{mg} / \mathrm{g}$ en novembre. Freeman (1986) a dosé 1,0 à plus de $7,0 \mathrm{mg} / \mathrm{g}$ dans Cladophora glomerata en Nouvelle Zélande.

Selon Gerloff (1966), la concentration en phosphore tissulaire limitante pour la croissance des végétaux aquatiques est $0,12 \%$ de la biomasse en poids sec soit $1,2 \mathrm{mg} / \mathrm{g}$ de poids sec. Chez Cladophora les valeurs limitantes rapportées par Freeman (1986) sont comprises entre 0,5 et $1,6 \mathrm{mg} / \mathrm{g}$ de poids sec.

Les teneurs moyennes que nous avons dosées sont supérieures à ces seuils : 2 à $3 \mathrm{mg} / \mathrm{g}$ dans les Potamots et 1,6 à $2,4 \mathrm{mg} / \mathrm{g}$ dans les Cladophores. Tout au plus peut-on déceler

\begin{tabular}{|l|c|c|c|c|}
\cline { 3 - 5 } \multicolumn{1}{c|}{} & \multirow{2}{*}{ P-PO $_{4}$} & \multirow{2}{*}{ P. Total } & \multicolumn{2}{c|}{$\mathrm{P}$ (mg/g de poids sec) } \\
\cline { 4 - 5 } & & & $P$. crispus & $P$. foliatus \\
\hline Tronçon 1A & $4 \mu \mathrm{g} /$ & $17 \mu \mathrm{g} /$ & $1,2-2,0$ & $1,5-2,0$ \\
\hline Tronçon 2 & $1150 \mu \mathrm{g} /$ & $1340 \mu \mathrm{g} /$ & $3,5-5,5$ & $4,5-8,0$ \\
\hline
\end{tabular}

Forsberg (1960) rapporte des teneurs en phosphore de 0,5 à $1,0 \mathrm{mg} / \mathrm{g}$ de poids sec chez les Characées en association avec des potamots dans des lacs. Sur les Charas, quelques échantillons plus pauvres en phosphore (par exemple à la station 33 en amont de la Luye).

Les orthophosphates (P-PO4) dans l'eau en Durance sont présents à la 
teneur de 5 à $12 \mu \mathrm{g} / \mathrm{l}$ en août et septembre, ils diminuent en novembre (<2 à $4 \mu \mathrm{g} /$ l) sans que le phosphore tissulaire ne s'appauvrisse puisque la valeur maximale est observée en novembre chez Groenlandia densa. La teneur en phosphore tissulaire ne suit pas obligatoirement les variations de la teneur en phosphore dans l'eau en raison du processus de stockage. D'autre part, la concentration mesurée dans l'eau n'est pas un bon indicateur de la charge apportée par les eaux usées en raison surtout de l'absorption rapide par les végétaux.

L'ensemble des données obtenues sur les tissus végétaux montre que le phosphore n'est pas, en l'état du système en 1985, limitant pour la croissance des macrophytes. D'autre part les dosages de phosphore dans le bassin d'Espinasses révèlent en août et septembre des teneurs de 6$20 \mu \mathrm{g} / \mathrm{l}$ en $\mathrm{P}_{-} \mathrm{PO}_{4}$ et $20-48 \mu \mathrm{g} / \mathrm{l}$ en phosphore total ce qui implique des teneurs non limitantes indépendamment des apports par les eaux usées.

II faut enfin souligner que le phosphore peut être prélevé par les macrophytes dans le sédiment.

La masse maximum de phosphore contenue dans la végétation atteint $86 \mathrm{~kg}$ en août 1985 dans le bassin d'Espinasses et $45 \mathrm{~kg}$ dans la Durance du pont d'Espinasses au pont de Rochebrune (tableau 16).

Si l'on admet un rejet de $4 \mathrm{~g}$ de $\mathrm{P}$ total par jour par habitant soit $1,46 \mathrm{~kg}$ par an, la végétation du bassin équivaut au rejet des eaux usées annuellement par 59 habitants et la végétation du tronçon de Durance étudié équivaut à 31 habitants.

Cette comparaison ne fournit qu'une approximation car il faudrait comparer la production biologique aux apports; elle montre cependant que l'ordre de grandeur des apports en phosphore suffisants pour produire une biomasse importante est très bas, vraisemblablement quelques centaines d'équivalent habitant. II semble donc difficile de limiter la végétation par une épuration du phosphore.

\section{7 - CONCLUSIONS.}

Les trois campagnes de mesures effectuées en août, septembre et novembre 1985 ont fourni des connaissances qualitatives et quantitatives sur les plantes macrophytes à l'aval de la retenue de Serre-Ponçon.

Le secteur étudié comporte le bassin de compensation d'Espinasses $(112,5 \mathrm{ha}, 2,9 \mathrm{~km}, 5,4 \mathrm{~m}$ de profondeur moyenne) et la Durance alimentée par le débit de $1,5 \mathrm{~m}^{3} / \mathrm{s}$ restitué au barrage d'Espinasses. En Durance des données quantitatives ont été recueillies jusqu'au pont de Rochebrune situé 2,8 km à l'aval d'Espinasses; des données complémentaires, semiquantitatives, ont été obtenues à l'aval jusqu'au pont de Tallard. La Durance est une succession de zones rapides peu ou pas envasées et de pools (petits bassins) très envasés.

La végétation est constituée de cinq taxons: les algues vertes Cladophora $s p$. et Spirogyra sp., des Characées, deux espèces de Pota- 
mogetonacées : Groenlandia densa et Potamogeton pectinatus.

Les conditions écologiques correspondant à la présence des principales catégories végétales sont rapportées dans le tableau 17.

Une évaluation quantitative a été réalisée dans le bassin de compensation et en Durance jusqu'au pont de Rochebrune. Les biomasses estivales obtenues sont proches des valeurs maximales citées dans la littérature; elles sont mentionnées dans le tableau ci-après : tante: $6-20 \mu \mathrm{g} / \mathrm{l}$ en $\mathrm{P}_{-} \mathrm{PO}_{4}, 20$ $48 \mu \mathrm{g} / \mathrm{l}$ en $\mathrm{P}$ total. Ces valeurs ne sont pas limitantes pour la croissance végétale.

- Durance : concentrations de 5 à $12 \mu \mathrm{g} /$ en $\mathrm{P}_{-} \mathrm{PO}_{4}$ en août et septembre et 2 à $4 \mu \mathrm{g} / \mathrm{l}$ en novembre. Les rejets d'eaux usées (égouts d'Espinasses, de Rémollon, de Gap par la Luye) apportent du phosphore qui disparaît à l'aval des émissaires. Les dosages de phosphore total dans la végétation donnent des valeurs qui ne sont pas limitantes d'après la littérature.

\begin{tabular}{|l|c|c|}
\cline { 2 - 3 } \multicolumn{1}{c|}{} & \multicolumn{2}{c|}{ Biomasse en août-septembre } \\
\cline { 2 - 3 } \multicolumn{1}{c|}{} & Poids sec en tonnes & Poids sec en kgha \\
\hline Bassin d'Espinasses & $70-95$ & $622-844$ \\
\hline Durance & $23-29$ & $1900-2300$ \\
\hline
\end{tabular}

Les Characées dominent quantitativement le peuplement $(51 \%$ de la biomasse en août, $83 \%$ en septembre). Les deux espèces de potamots représentent $45 \%$ de la biomasse en août et $14 \%$ en septembre. Les algues vertes restent relativement peu abondantes; toutefois elles décroissent moins que les autres catégories en fin novembre où elles représentent environ $5 \%$ de la biomasse dans le secteur étudié.

Des analyses de nutriments dans l'eau, réalisées lors des campagnes de mesures, montrent une évolution du phosphore qui peut être schématisée ainsi :

- bassin d'Espinasses: concentration au turbinage des eaux de Serre-Ponçon relativement cons-
Contrairement au maximum de biomasse qui intervient fin septembre, le maximum de phosphore dans la végétation se situe à la fin août : $86 \mathrm{~kg}$ dans le bassin de compensation et $45 \mathrm{~kg}$ en Durance. Cela représente le rejet annuel de quelques dizaines d'équivalents habitants ( $4 \mathrm{~g} \mathrm{P} /$ jour).

De ces éléments se dégage la conclusion que le phosphore apporté par l'eau de Serre-Ponçon, bien qu'en faible concentration, n'est pas limitant pour la croissance végétale en été dans le bassin de compensation. En Durance interviennent des apports supplémentaires par les eaux usées.

Les mesures de qualité d'eau réalisées par deux stations automatiques situées en Durance mettent en évidence l'influence de la photosynthèse 


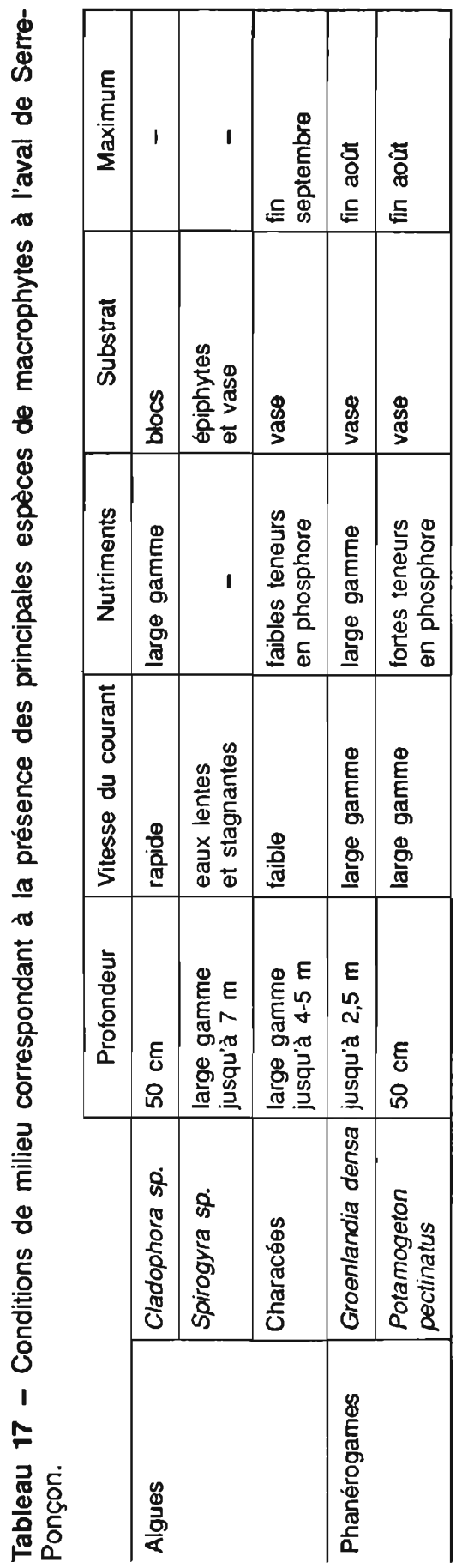


et de la respiration végétale sur le bilan d'oxygène dissous et le $\mathrm{pH}$. Ces paramètres présentent des variations nycthémérales d'amplitude non négligeable: $3,0 \mathrm{mg} / \mathrm{l}$ pour l'oxygène en septembre. Toutefois, la teneur en oxygène dissous est restée supérieure ou égale à $7,8 \mathrm{mg} / \mathrm{l}$ en moyenne mensuelle, le minimum n'étant pas inférieur à $6,0 \mathrm{mg} / \mathrm{l}$.

\section{REFERENCES}

Anderson M.G., 1978. Distribution and production of Sago pondweed (Potamogeton pectinatus Lo) on a northern prairie Marsh -Ecology, 59, (1): 154160.

Anonyme 1974. Qualité des eaux de la Dordogne - Approche de l'influence des réservoirs - Agence Financière de Bassin Adour-Garonne.

Anonyme 1982. Etude hydrobiologique de la Dordogne - Rapport à E.O.F., CEMAGREF section Qual. Eaux Pech. Pisc., Bordeaux : 265 p.

Barko J.W. et Smart R.M., 1980. Mobilisation of sediment phosphorus by submersed freshwater macrophytes Freshwater biol. 10 : 229-238.

Blindow.l, 1988. Phosphorus toxicity in Chara. Aquatic Botany, 32, 393-395.

Bristow J.M. et Whitcombe M., 1971. The role of root in the nutrition of aquatic vascular plants - Amer. J. Bat. 58, (1) : 8-13.

Carigan R, et Ralff J., 1980. Phosphorus sources for aquatic weeds: water or sediment? - Science 207 : 987-989.

Champeau A. et Coll, 1980. Etude hydrobiologique de la retenue de Serre-Ponçon (Hautes-Alpes) : $1^{\text {er }}$ cycle annuel Rapport E.D.F. E.2271 : 125 p.

Corillon R., 1957. Les Charophycées de France el d'Europe occidentale - Bull. Soc. Sc de Bretagne, Fasc. Hors série, $n^{\circ}$ I, II: 7-499.
Dale H.M., 1986. Temperature and light: the determining factors in maximum depth distribution of aquatic macrophytes in Ontario, Canada - Hydrobiologia $133: 73-77$.

Denny P., 1972. Sites of nutrient absorption in aquatic macrophytes - J. of Ecology, $60: 819-829$.

Descamps H., Capblancq J., Casanova H. et Tourenq J.N., 1975. Hydrobiology of some regulated rivers in the south-west of France - In Ward J.V. et Standford J.A. Ed - The ecology of regulated streams, Plenum press, New-York.

Dykyjova D., Kosanova A., Husak S. et Sladeckova A., 1985. Macrophytes and water pollution of the Zlata Stoka (Golden canal), Trebon biosphère Reserve, Czechoslovakia - Arch. Hydrobiol. 105 (1) : 31-58.

Forsberg C., 1960. Subaquatic macrovegetation in Osbysjon, Djursholm -Oikos 11 (2) : 183-199.

Forsberg C., 1964. Phosphorus, a maximum factor in the growth of characeaeNature 201 : 517-518.

Freeman M.C., 1986. The role of nitrogen and phosphorus in the development of Cladophora glomerata Kutzing in the Manawatur river, New Zealand Hydrobiologia 131 : 23-30.

Gerloff G., 1966. Evaluating nutrient supplies for the growth of aquatic plants Academy of Sciences, Washington DC pp. 537-555 - In M. Wright A.J. McDonnell 1986.

Haslam S.M., 1975. River vegetation and pollution - In R.D. Hey et T.D. Davies (Eds.) : Science, Technology and Environmental Management: 137-143.

Hill A.H., 1982. Phosphorus and Major cation mass balances for two rivers during low summer flows - Freshwater biol. 12 : 293-304.

Ho Y.B., 1979. Inorganic mineral nutrient level studies on Potamogeton pectinatus L. and Enteromorpha prolifera in Torfar Loch, Scotland - Hydrobiologia 62 (I) : 1-15. 
Jorgensen S.E., 1979. Handbook of environmental data and ecological parameters. International Society for Ecological Modelling : $161 \mathrm{p}$.

Khalanski M., Bonnet M. et Grégoire A., 1987. Rapport E.D.F. DER. HE/3287.05 : 58 p., 8 annexes.

Kohler A., 1971. Zur Okologie submerser Gefa - Macrophyten in Flie gewassern - Ber. Dtsch. Ges. Bd, 84 (11) : 713 720.

Lachavanne J.B., 1985. The influence of accelerated eutrophication on the macrophytes of Swiss lakes: Abondance and distribution - Verh. Internat. Verein. Limnol. 22 : 2 950-2 955.

Lachavanne J.B., Juge R. et Noetzlin A., 1986. Evolution de la végétation aquatique des rives génoises du Leman (1972-1984) - In Assoc. Fr. Limmol., Actes congr. Natl. Thonon-lesBains 30.

Mattes H. et Kreeb K., 1974. Die Nettophotosynthese von Wasserpflanzen, insbesondere Potamogeton densus, als indicator fûr die Verunreimigumg von Gewassern - Angew. Botanik, 48 : 287-297.

Meyer J.L., 1979. The role of sediments and bryophytes in phosphorus dynamics in a headwater stream ecosystem Limnol. Oceanogr. 24 : 365-375.

Nisbet M. et Verneaux J., 1970. Composantes chimiques des eaux courantes. Discussion et proposition de classes en tant que bases d'interprétation des analyses chimiques - Annls Limnol. 6 (2) : 161-190.

Ozimek T., 1978. Effect of municipal sewage on the submerged macrophytes of a lake littoral - Ekol. pol. 26 (1) : 339.

Pereira-Ramos E., 1981. The ecological role of characeae in the lake littoral Ekol. Pol., 29 (2) : 167-209.

Pip E., 1984. Ecogeographical tolerance range variation in aquatic macrophytes - Hydrobiologia, 108 : 37-48.
Sculthorpe C.D., 1967. The biology of aquatic vascular plants -610 p. - Edward Arnold - London.

Stake E., 1968. Higher vegetation and phosphorus in a small stream in central Sweden - Schweiz A. Hydrol. 30 (2) : 353-373.

Valet G., 1959. Monographie des cladophora de la région de Sète - Université de Montpellier - Faculté des Sciences, institut de botanique, Travaux II : $90 \mathrm{p}$.

Vermaak J.F., Swanzpoel J.H. et Schoonbee H.J., 1981. The phosphorus cycle in Germiston lake. 1. Investigational objectives and aspect of the limnology of the lake water S.A., 7 (3) : 160-165.

Vermaak J.F., Swanzpoel J.H. et Schoonbee H.J., 1982. The phosphorus cycle in Germiston lake. 2. The in vitro and in vivo absorption of $32 \mathrm{P}$ by Potamogeton pectinatus L. Water S.A., 8 (1) : 52-57.

Vermaak J.F., Swanzpoel J.H. et Schoonbee H.J., 1982. The phosphorus cycle in Germiston lake. 3. Seasonal patterns in the absorpion, translocation and release of phosphorus by Potamogeton pectinatus. L. Water S.A., 8 (3) : 138-141.

Westlake D.F., 1973. Aquatic macrophytes in river, a review. - Pol. Arch. Hydrobiol., $20: 31-40$.

Whitford L.A. et Sclucmacher G.J., 1961. Effect of current on mineral uptake and respiration by a freshwater alga. - Limmol. Oceanogr. $6:$ 423-425.

Wong S.L., Clark B., Kurby M. et Koscium R.F., 1978. Water temperature fluctuation and seasonal periodicity of Cladophora and Potamogeton in shallow rivers - J. Fish. Rəs. Board Can., 35 : 866-870.

Wright M. et McDonnell A.J., 1986. Macrophyte growth in shallow streams: field investigations. - Journal of Environmental Engineering, Vol. 112, $n^{\circ} 5$, october. 